Accepted refereed manuscript of:

Whiten A, Caldwell CA \& Mesoudi A (2016) Cultural diffusion in humans and other animals, Current Opinion in Psychology, 8, pp. 15-21.

DOI: $\underline{10.1016 / j . c o p s y c .2015 .09 .002}$

(c) 2016, Elsevier. Licensed under the Creative Commons AttributionNonCommercial-NoDerivatives 4.0 International

http://creativecommons.org/licenses/by-nc-nd/4.0/ 


\section{Cultural diffusion in humans and other animals}

Andrew Whiten ${ }^{1}$, Christine A Caldwell ${ }^{2}$ and Alex Mesoudi ${ }^{3}$

Recent years have seen an enormous expansion and progress in studies of the cultural diffusion processes through which behaviour patterns, ideas and artifacts are transmitted within and between generations of humans and other animals. The first of two main approaches focuses on identifying, tracing and understanding cultural diffusion as it naturally occurs, an essential foundation to any science of culture. This endeavor has been enriched in recent years by sophisticated statistical methods and surprising new discoveries particularly in humans, other primates and cetaceans. This work has been complemented by a growing corpus of powerful, purpose-designed cultural diffusion experiments with captive and natural populations, that have facilitated the rigorous identification and analysis of cultural diffusion in species from insects to humans.

\section{Addresses}

${ }^{1}$ Centre for Social Learning and Cognitive Evolution, School of Psychology \& Neuroscience, University of St Andrews, St Andrews KY16 9JP, UK.

2 Psychology, School of Natural Sciences, University of Stirling, Stirling FK9 4LA, UK

${ }^{3}$ Human Biological and Cultural Evolution Group, Department of Biosciences, College of Life and Environmental Sciences, University of Exeter Cornwall Campus, Penryn, Cornwall TR10 9FE, UK

Corresponding author: Whiten, A (a.whiten@st-and.ac.auk)

This review comes from a themed issue on Culture 
Highlights

- Decades-long field studies yield evidence of widespread animal cultures

- New statistical approaches track the cultural diffusion of animal innovations

- From insects to primates, diffusion experiments rigorously confirm cultural diffusion

- Sophisticated statistical approaches trace human cultural diffusion on micro and macro scales

- Diffusion experiments analyze the what, who, when and how of human transmission 


\section{Introduction}

At the core of the phenomenon of culture, whether in humans or non-human animals (henceforth 'animals'), are processes whereby entities including behaviour patterns, ideas and artifact designs spread between or within generations, maintaining some recognizable consistency of form. Such entities are often described as 'traditions', and the underlying social learning processes as 'cultural diffusion' or 'cultural transmission' [1-4]. The field has expanded enormously in recent years, often driven by methodological advances and maturing long-term field studies, generating multiple major advances [1-9].

These have often highlighted increasingly strong links between animal and human phenomena $[1,2,8,9]$. However, the unique aspects of human culture remain sufficiently distinctive that we review animal and human studies in turn.

\section{Cultural Diffusion in Animal Field Studies}

As long term field studies have matured in recent decades, putative cultural differences between subpopulations have been delineated, particularly in avian, cetacean and primate species [8-10]. These typically reflect stable patterns, so opportunities to record the actual diffusion of spontaneous innovations are rare. However, cases have begun to be published.

The cultural basis of some have been identified through new techniques of 'network-based diffusion analysis' (NBDA), in which diffusion following the lines of social networks implicates transmission via social learning from close associates $[11,12]$. Pioneering examples include tracing of the diffusion of 'lob-tail feeding' from its first occurrence in humpback whales, to its spread along networks among 653 whales over 27 years, based on over 73,000 observations [13: see Figure 1]. At the other extreme, the invention and diffusion of using moss as a tool for sponging water by wild chimpanzees was tracked across a sequence of just days by a variant of this networkbased technique $[14,15]$.

\footnotetext{
*** insert Figure 1 about here $* * *$
} 
Diffusion has also been inferred from inter-group transfers. A recent example among chimpanzees is the spread of a novel form of ant-fishing from one community to its neighbours [16]. By contrast, female chimpanzees in the Tai Forest moving to a neighbouring community were shown to conform to local preferences in the selection of hammer materials for nut-cracking [17]. A major question is thus what throws the switch between incomers conforming, and incomers' behaviour instead being adopted by residents [15]. A recent striking example of the conformist alternative in the vocal domain is immigrant chimpanzees converging on local 'referential' vocalization styles that signal high-quality foraging options [18].

A dramatic contrast to the conservatism suggested by many studies of animal culture also comes from the vocal domain. The songs of humpback whales are similar across large areas of ocean, yet may change and diffuse rapidly, constituting 'cultural revolutions' [19]. Recently such changes have been observed to diffuse across the Pacific ocean like 'cultural ripples' [20]. Songs originating near Australia in 1998 and 2002 spread to French Polynesia by 2001 and 2004 respectively, being recorded at four intermediate locations in between.

\section{Animal Cultural Diffusion Experiments}

It is often difficult to confidently identify a causal role for social learning in observational field studies, whereas this is precisely what controlled experiments can do. Such experimental studies of animal social learning have a history of over a century, but for a long time involved only single subjects observing a single model. Relevance to the 'macro' scale of culture required a different approach, which later developed in three main forms [3-4]: (i) diffusion (or transmission) chains, that begin with a trained model and then follow a sequence in which observers successively become models for a next observer in the chain; (ii) open diffusion, where the means by which traits spread from trained models or spontaneous innovators is left open; and (iii) replacement designs where, over successive 'generations', some group members are replaced with naïve incomers. These designs each tell us something different and are complementary.

Whiten and Mesoudi [3] reviewed 33 animal diffusion experiments conducted from 1972 to 2008, spanning fish, birds, primates and rodents. The rate of such studies has since escalated, such that supplementary table S1 lists a further 30 experiments 
2009-2015, extending the taxonomic coverage to insects as well [21]. There is a welcome increase in field experiments, from $3 / 33$ in 2008 to $14 / 30$ now. We cannot comprehensively review these studies here but Table S1 offers terse summaries of each study's contribution. Advances on several fronts deserve mention.

A first cluster of advances are methodological. The 2008 review [3] systematized the 33 experiments reviewed into a matrix structured by the three kinds of experiments outlined above, and seven different contrasts among experimental and control conditions. Studies were found to span as many as 15 of the resulting 21 cells in the matrix. It is noticeable that 27 of the 30 more recent studies have converged on one of the three approaches, open diffusion. This might suggest a developing view that this is the most valuable of the three, arguably representing many natural situations, such as when an individual with a novel skill immigrates into a new group. However it may simply be that diffusion chains (just 3/33 studies) can be hard to engineer in animals that have the potential for aggression between pairs put together, such as chimpanzees [22]. The open diffusion experiments are now commonly coupled with the strongest condition contrasts advocated in ref [3], which have two different behavioural options seeded in two or more groups (Figure 2), ideally with the addition of a no-model control condition.

$* * *$ insert Figure 2 about here $* * *$

Perhaps most surprising is the absence of replacement designs in the present table, because these also represent a common scenario in real world animal groups shaped by immigrations, emigrations, births and deaths. However a new approach in some studies is to incorporate multiple models. At one extreme, all existing members of whole groups of monkeys were trained in food preferences, followed by testing of maturing naïve infants and immigrants with opposing preferences [23]. This revealed potent social learning effects [23] echoing the spontaneous conformity in chimpanzees noted above [17]. Similar findings have been observed in species as diverse as great tits [24] and drosophila [21]. Several field studies introducing only single models found more fragile social learning effects, so the multiple-model approach - which is 
consistent with other evidence for conformity-to-majority effects in animals [25] - may repay more research in future.

Other pioneering methods advancing our understanding have included extending the use of video models to field conditions [26] and combining social network analyses like NBDA with diffusion experiments [24, 27]. Whilst as in 2008 most of the animal social diffusion experiments were addressing only the (fundamental) question of the capacity for cultural diffusion in the species and context studied, these newer studies analyzing social networks illustrate a shift to tackling the underlying decision rules. For example, squirrel monkeys central in the social network tended to be the first to participate in the diffusion of new behavioural variants [27] and chimpanzees preferentially learned from high ranking and knowledgeable group members [28].

\section{Cultural Diffusion in 'Real-world' Human Populations}

Efforts to trace the diffusion and cultural evolution of human technologies, languages and other cultural phenomena have a history of well over a century, including historical, archaeological, anthropological and sociological studies spanning the whole gamut of the humanities and social sciences. Recent advances have been generated by the application of increasingly sophisticated statistical methodologies, in some cases derived from other scientific domains like evolutionary biology $[1,2,6,7,29,30]$.

These have taken perspectives ranging from the 'micro', concerned with observable diffusion processes at the inter-personal level, to 'macro' analyses spanning large geographic areas and/or time scales that may extend to centuries. A pioneering recent example of relatively 'micro' analysis charted transmission networks for particular skills like fishing and herbal medicine in traditional village societies, finding a shift from learning from parents in early childhood to selective learning from relevant experts with age [31].

A longer-term perspective comes from archaeology, where the focus is on directly tracing records of diffusion over often large regions and timeframes. For example a recent study found that the S-shaped curve characterizing the slow, then accelerating, then plateauing diffusion of innovations identified in many more modern studies is also observed in prehistoric hunter-gatherer societies, exemplified in this case by the diffusion of pottery designs, but spread over several centuries across the western 
Great Basin of America [32]. Shennan [33] and Lycett [34] offer up-to-date reviews of research of this kind and the value of an evolutionarily-informed approach to the archaeological analysis of cultural diffusions.

Other approaches search for the signatures of cultural phylogeny in present day cultural variation. One recent study targeted socio-political structures across SE Asia and the Pacific, finding evidence for the cultural diffusion and evolution of four escalating levels of political structure across this vast region [35]. Arguably the biggest strides have been made in cultural phylogenetic studies of language evolution, for example tracing language diffusion and evolution accompanying the migration of populations across this same Pacific region [36] and others [37]. On the grandest scale, such approaches based on over 500 languages have generated evidence for an African origin of all today's languages [38].

\section{Human Cultural Diffusion Experiments}

Parallel to their review of animal studies [3], Mesoudi and Whiten reviewed 34 adult human diffusion experiments extant by 2008 [4]. Table S2 lists 61 more studies of this kind published since that review, confirming the explosion of interest and achievement in this area. Whilst only a handful of the animal experiments go beyond checking the capacity of the species concerned for cultural transmission, this can be assumed for humans and the human experiments go further in asking a variety of questions about the "what, who, when and how" of diffusion, extending a trend already apparent in 2008 [4: see Figure 3 for an example]. Perhaps the closest comparison between the two sets of studies comes from the addition of child experiments in the new human corpus, often following a similar design and rationale to the primate experiments and in some cases facilitating direct comparison [39-40].

*** plcase insert Figure 3 about here ***

The growing human literature defies comprehensive review in the space available here. Instead, we highlight some welcome developments that strike us. First, in addition to the incorporation of children [39-40 and Table S2], participation has been extended to overdue cross-cultural comparisons, finding more use of social learning in 
East Asian samples known for their more collectivist attitudes [41]; moreover in several studies, marked individual differences in reliance on different social learning strategies and even in reliance on social learning per se, have been identified [42-44]. The content of what is transmitted has further diversified, ranging from the technical like tool use [39-40] and even flint-knapping [45] to the social, such as stereotypes [46] (see also Kirby, in this issue, on language transmission and evolution). By 2008, the "how" question concerning diffusion remained little tackled, but more recent studies dissect social learning into categories like imitation [47-49] and teaching [45]. Studies have also explored the effects of the size and nature of the pool of models from whom participants can learn [50-53].

In the growing suite of diffusion experiments examining the factors affecting cumulative cultural evolution in laboratory micro-societies [47-53], accumulation is generally expected to involve an increase in complexity or sophistication in the phenomena at hand, such as escalating heights of constructed towers [47]. However, the new corpus of diffusion experiments has been widened by several studies of communicative conventions, ranging from artificial languages [54] to graphical images [55] and here, the principal change along diffusion chains instead typically involves some kind of simplification or stylization that facilitates remembering, communication and transmission. A similar effect is the focus of a single animal study, to date [56].

\section{Conclusions, Further Reading and Future Prospects}

The field is clearly in robust health and displaying a growing richness in scope and understanding of the cultural transmission processes active in our own species and others. Two recent edited volumes incorporate a diversity of reviews by many leaders in the subject and are recommended to readers who wish to further explore the scope and potential of recent studies of cultural diffusion $[6,57]$.

Future prospects for this ficld arc accordingly expansive and as our review suggests, now have at their disposal an array of exciting new methodological approaches to exploit and elaborate on. However whilst as noted there is a welcome expansion of the power of experiments into wild and natural context in animal studies, laboratory studies dominate the human arena and there is much scope for researchers on humans to emulate experimental animal ethologists in creating more ecologically valid 
'field experiments' [39-40]. More generally, it must be remembered that there are two essential components of cultural evolution; innovation and dissemination. Experimental seeding of the kind we have reviewed is perfect for elucidating the latter but by its very nature excludes the innovation element. Advancing ecological validity by bringing innovation into a more comprehensive experimental science of culture is a needed but challenging prospect.

\section{Acknowledgements}

AW was supported by grant ID40128 from the John Templeton Foundation during the writing of this paper. 


\section{References}

[1] Whiten A, Hinde RA, Laland KN, Stringer CB: Culture evolves. Philos Trans $R$ Soc Lond B Biol Sci 2011, 366:938-948.

[2] Hoppitt W, Laland KN: Social Learning: An Introduction to Mechanisms, Methods and Models. Princeton University Press; 2013.

[3] Whiten A, Mesoudi A: Establishing an experimental science of culture: animal social diffusion experiments. Philos Trans R Soc Lond B Biol Sci 2008, 363:34773488.

[4] Mesoudi A, Whiten A: The multiple roles of cultural transmission experiements in understanding human cultural evolution. Philos Trans $R$ Soc Lond B Biol Sci 2008, 363:3489-3501.

[5] Laland KN, Galef B: The Question of Animal Culture. Cambridge: Harvard University Press; 2009.

[6] Mesoudi A: Cultural Evolution. Chicago, IL: Univ. Chicago Press; 2011.

[7] Richerson P, Christiansen MH: Cultural Evolution: Society, Technology, Language and Religion. Cambridge: MIT Press; 2013.

[8] Whitehead H, Rendell L: The Cultural Lives of Whales and Dolphins. Chicago: University of Chicago Press; 2015.

[9] Whiten A: The scope of culture in chimpanzees, humans and ancestral apes. Philos Trans R Soc Lond B Biol Sci 2011, 366:997-1007.

[10] Holzhaider JC, Hunt GR, Gray RD: Social learning in New Caledonian crows. Learn Behav 2010, 38:206-219.

[11] Franz M, Nunn CL: Network-based diffusion analysis: a new method for detecting social learning. Proc Biol Sci 2009, 276:1829-1836.

[12] Hoppitt W, Boogert NJ, Laland KN: Detecting social transmission in networks. $J$ Theor Biol 2010, 263:544-555.

**[13] Allen J, Weinrich M, Hoppitt W, Rendell L: Network-based diffusion analysis reveals cultural transmission of lobtail feeding in humpback whales. Science 2013, 340:485-488.

Uses 27 years of observation to track the spread of a foraging innovation from its beginnings; its spread along independently identified social networks implicates social learning, making it a compelling case of cultural diffusion. 
**[14] Hobaiter C, Poisot T, Zuberbuehler K, Hoppitt W, Gruber G: Social network analysis shows direct evidence for social transmission of tool use in wild chimpanzees. Plos Biology 2014, 12:1-12.

Exploited a rare occurrence of an innovation in tool use (using moss to make sponges to obtain water from a pool) to track its spread across the group, following social network lines and confirming it as a case of cultural diffusion.

[15] Whiten A: Incipient tradition in chimpanzees. Nature 2014, 514:178-179.

[16] O'Malley RC, Wallauer W, Goodall $\mathrm{J}$ : The appearance and spread of ant fishing among Kasekela chimpanzees of Gombe. Current Anthropology 2012, 53:650670

**[17] Luncz LV, Boesch C: Tradition over trend: Neighboring chimpanzee communities maintain differences in cultural behaviour despite frequent immigration of adult females. Am J Primatol 2014, 76:649-657.

Shows that differences in preferred natural hammer materials between neighboring communities that are not explained by available materials are adopted by females who typically migrate to other communities once sexually mature. The differences thus appear to represent local cultural norms that diffuse to immigrants, hence maintain intercommunity differences.

*[18] Watson SK, Townsend SW, Schel AM, Wilke C, Wallace EK, Cheng L, West V, Slocombe KE: Vocal learning in the functionally referential food grunts of chimpanzees. Curr Biol 2015, 25:495-499.

Chimpanzees employ grunts described as functionally referential when they signal differences in the quality of food sources discovered. A group of chimpanzees introduced into a Zoo community initially used recognisably different vocalisations for this purpose than the residents, but over time converged on the local vocal conventions.

[19] Noad MJ, Cato DH, Bryden MM, Jenner MN, Jenner KCS: Cultural revolution in whale songs. Nature 2000, 408.

[20] Garland EC, Goldizen AW, Rekdahl ML, Constantine R, Garrigue C, Hauser ND, Poole MM, Robbins J, Noad MJ: Dynamic horizontal cultural transmission of humpback whale song at the ocean basin scale. Curr Biol 2011, 21:687-691. 
[21] Battesti M, Moreno C, Joly D, Mery F: Spread of social information and dynamics of social transmission within Drosophila groups. Curr Biol 2012, 22:309-313.

[22] Horner V, Whiten A, Flynn E, de Waal FB: Faithful replication of foraging techniques along cultural transmission chains by chimpanzees and children. Proc Natl Acad Sci U S A 2006, 103:13878-13883.

**[23] van de Waal E, Borgeaud C, Whiten A: Potent social learning and conformity shape a wild primate's foraging decisions. Science 2013, 340:483-485.

Took the novel approach of training whole groups (instead of the usual single, seeded individual) to prefer one of two feeding options and investigated the effect on naïve infants and immigrant males. All infants adopted the preference of their mothers, and most surprisingly, males trained on one option and moving to a group with the alternative preference, immediately conformed to that local norm.

**[24] Aplin LM, Farine DR, Morand-Ferron J, Cockburn A, Thornton A, Sheldon BC:

Experimentally induced innovations lead to persistent culture via conformity in wild birds. Nature 2015, 518:538-541.

Seeding different communities of wild great tits with alternative foraging behaviours generated consistent differences that endured across seasons. As in Ref [23], many immigrants conformed to the local norms: these two studies are the first to demonstrate this effect in wild primates and birds respectively.

[25] Claidiere N, Whiten A: Integrating the study of conformity and culture in humans and nonhuman animals. Psychol Bull 2012, 138:126-145.

[26] Gunhold T, Whiten A, Bugnyar T: Video demonstrations seed alternative problem-solving techniques in wild common marmosets. Biol Lett 2014, 10.

[27] Claidiere N, Messer EJ, Hoppitt W, Whiten A: Diffusion dynamics of socially learned foraging techniques in squirrel monkeys. Curr Biol 2013, 23:1251-1255. *[28] Kendal R, Hopper LM, Whiten A, Brosnan SF, Lambeth SP, Schapiro SJ, Hoppitt W: Chimpanzees copy dominant and knowledgeable individuals: implications for cultural diversity. Evolution and Human Behaviour 2015, 36:65-72. Combined two-action diffusion experiments with statistical modelling to reveal evidence for preferential learning from high-ranked and knowledgeable (successful) models, and biases towards social learning when of low rank or when uncertain. 
[29] Mesoudi A: Cultural evolution: Integrating Psychology, Evolution and Culture. Curr Opin Psychol 2016, this issue.

[30] Jordan P: Technology as Human Social Tradition. California: University of California Press; 2015

[31] Henrich J, Broesch J: On the nature of cultural transmission networks: evidence from Fijian villages for adaptive learning biases. Philos Trans $R$ Soc Lond B Biol Sci 2011, 366:1139-1148.

[32] Eerkens JW, Lipo CP: A tale of two technologies: Prehistoric diffusion of pottery innovations among hunter-gatherers. Journal of Anthropological Archaeology 2014, 35:23-31.

[33] Shennan S: Long-term trajectories of technological change. In Cultural Evolution: Society, Technology, Language and Religion. Edited by Richerson P, Christiansen MH. MIT Press; 2013:143-155.

[34] Lycett SJ: Cultural evolutionary approaches to artifact variation over time and space: basis, progress, and prospects. J Archaeol Sci 2015, 56:21-31.

[35] Currie T, Mace R: Mode and tempo in the socio-political organization: reconciling 'Darwinian' and 'Spencerian' evolutionary approaches in anothropology. Philos Trans R Soc Lond B Biol Sci 2011, 366:1108-1117.

[36] Gray RD, Atkinson QD, Greenhill SJ: Language evolution and human history: what a difference a date makes. Philos Trans R Soc Lond B Biol Sci 2011, 366:1090-1100.

[37] Bouckaert R, Lemey P, Dunn M, Greenhill SJ, Alekseyenko AV, Drummond AJ, Gray RD, Suchard MA, Atkinson QD: Mapping the origins and expansion of the Indo-European language family. Science 2012, 337:957-960.

[38] Atkinson QD: Phonemic diversity supports a serial founder effect model of language expansion from Africa. Science 2011, 332:346-349.

[39] Whiten A, Flynn E: The transmission and evolution of experimental microcultures in groups of young children. Dev Psychol 2010, 46:1694-1709.

[40] Flynn E, Whiten A: Experimental "microcultures" in young children: Identifying biographic, cognitive, and social predictors of information transmission. Child Development 2012, 83:911-925. 
*[41] Mesoudi A, Chang L, Murray K, Lu HJ: Higher frequency of social learning in China than in the West shows cultural variation in the dynamics of cultural evolution. Proc Biol Sci 2015, 282:20142209.

This study showed that participants from mainland China showed more frequent social learning in a computer-based artifact design task than White British participants in the UK, as well as Chinese immigrants to the UK and participants from Hong Kong. This suggests broader cultural variation in human's use of social information.

[42] van den Berg P, Molleman L, Weissing FJ: Focus on the success of others leads to selfish behaviour. Proceedings of the National Academy of Sciences 2015, 112:2912-2917.

[43] Toelch U, Bruce MJ, Newson L, Richerson PJ, Reader SM: Individual consistency and flexibility in human social information use. Proc Biol Sci 2014, 281:20132864.

[44] Cook JL, den Ouden HEM, Heyes CM, Cools R: The social dominance paradox. Curr Biol 2014, 24:2812-2816.

[45] Morgan TJ, Uomini NT, Rendell LE, Chouinard-Thuly L, Street SE, Lewis HM, Cross CP, Evans C, Kearney R, de la Torre I, et al.: Experimental evidence for the co-evolution of hominin tool-making teaching and language. Nat Commun 2015, 6:6029.

[46] Kashima Y, Lyons A, Clark A: The maintenance of cultural stereotypes in the conversational retelling of narratives. Asian J Soc Psychol 2013, 16:60-70.

[47] Caldwell CA, Millen AE: Social learning mechanisms and cumulative cultural evolution. Is imitation necessary? Psychol Sci 2009, 20:1478-1483.

[48] Wasielewski H: Imitation is necessary for cumulative cultural evolution in an unfamiliar, opaque task. Human Nature, 2014, 25:161-179.

[49] Derex M, Godelle B, Raymond M: Social learners require process information to outperform individual learners. Evolution 2012, 67:688-697.

[50] Muthukrishna M, Shulman BW, Vasilescu V, Henrich J: Sociality influences cultural complexity. Proc Biol Sci 2014, 281:20132511.

[51] Kempe M, Mesoudi A: An experimental demonstration of the effect of group size on cultural accumulation. Evolution and Human Behaviour 2014, 35:285-290. 
*[52] Derex M, Beugin MP, Godelle B, Raymond M: Experimental evidence for the influence of group size on cultural complexity. Nature 2013, 503:389-391.

This study used a computer-based fishing task to show that larger groups can maintain more complex cultural traits than smaller groups, illustrating the importance of demography on cumulative cultural evolution. See also refs 49 and 50.

[53] Caldwell CA, Millen AE: Human cumulative culture in the laboratory: effects of (micro) population size. Learn Behav, 2010, 38:310-318.

[54] Smith K, Wonnacott E: Eliminating unpredictable variation through iterated learning. Cognition 2010, 116:444-449.

[55] Caldwell CA, Smith K: Cultural evolution and perpetuation of arbitrary communicative conventions in cxperimental microsocieties. Plos One 2012, 7.

[56] Claidiere N, Smith K, Kirby S, Fagot J: Cultural evolution of systematically structured behaviour in a non-human primate. Proc Biol Sci 2014, 281: 20141541.

[57] Whiten A, Hinde RA, Stringer CB, Laland KN (Eds): Culture Evolves. Oxford University Press; 2012. 
Figure captions

Figure 1. Diffusion of lob-tail foraging in a social network of humpback whales. Individuals close to the centre of the network plot are well connected with the others; blue nodes are those observed lob-tail feeding at least 20 times, red nodes those never observed lob-tail feeding. After Allen et al. 2013.

Figure 2. An 'open' diffusion experiment with wild vervet monkeys.

(a,b) Vervet monkeys opening an artificial fruit ('vervetable') by alternative methods of lift versus slide, seeded in trained models in different groups. (c) Inferred information flow through group AK, seeded with 'lift' approach. Each column represents one vervet and each row represents a session day (numbered), with entries diagonally left to right expressing each individual's first opening techniques on the relevant session: on left in white box, number of lifts; on right in grey box, number of slides. The bold frame indicates the most common action in each case. Arrows tracked backwards show whom an individual had been in a position to observe before starting to open vervetables, and the relative numbers of 'lift' versus 'slide' they were thus inferred to have witnessed before their first successful opening, so arrow heads indicate inferred information flow. Numbers across the bottom of the diagram show the total frequencies of alternative actions in the whole series of sessions. Stars indicate whether the first action done was lift or slide. After van de Waal et al. 2015, cited in Table S1.

Figure 3. Cultural differences transmitted along laboratory diffusion chains: spaghetti towers created by participants in Caldwell \& Millen (2010a, cited in Table S2). Each row displays the complete set of towers that were produced by one chain of participants. The towers are ordered from left to right, in the order in which they were produced (i.e. the first participant's tower is on the far left, and the tenth on the far right).

Supplementary Tables S1, S2 - are in separate files. 


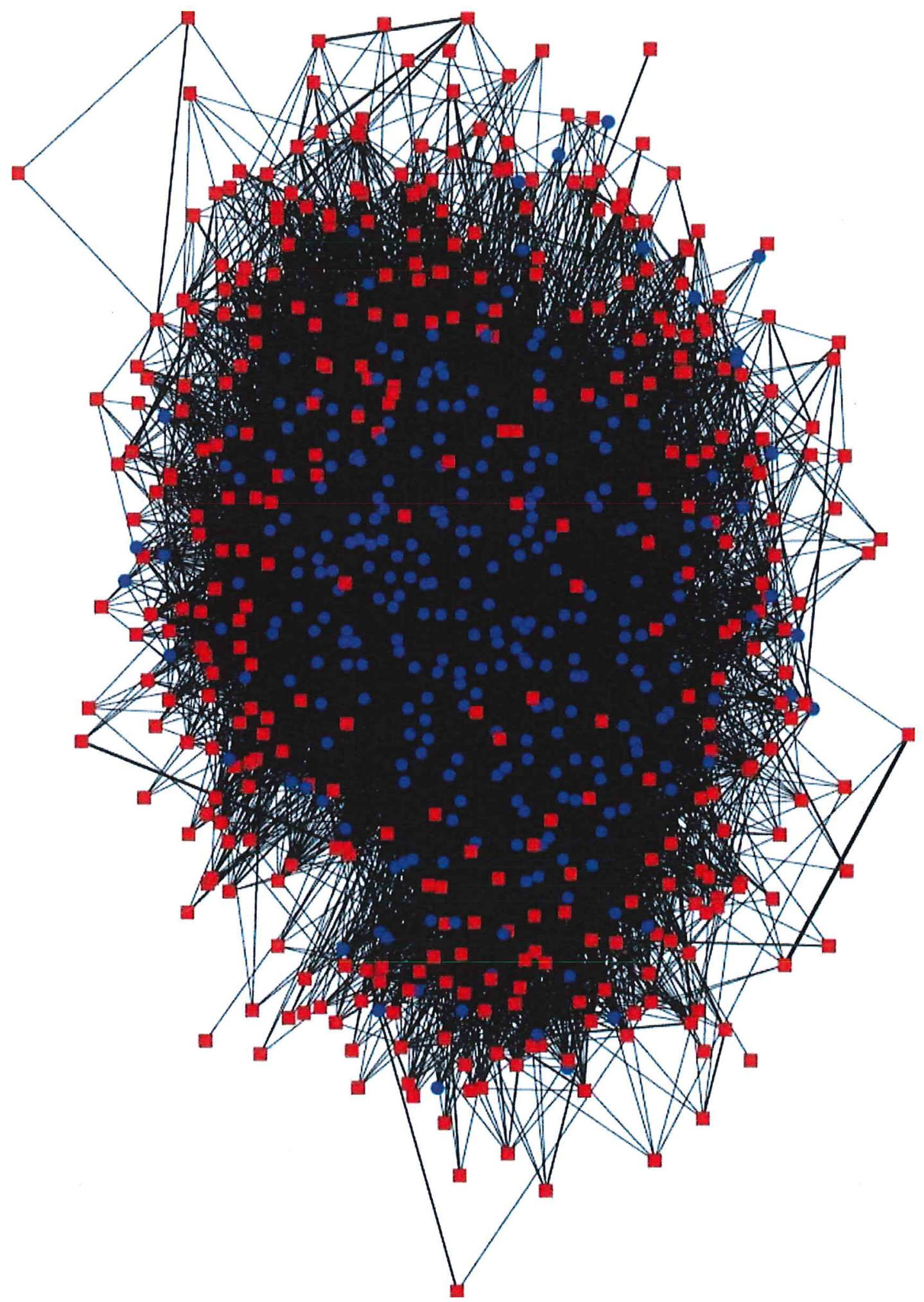



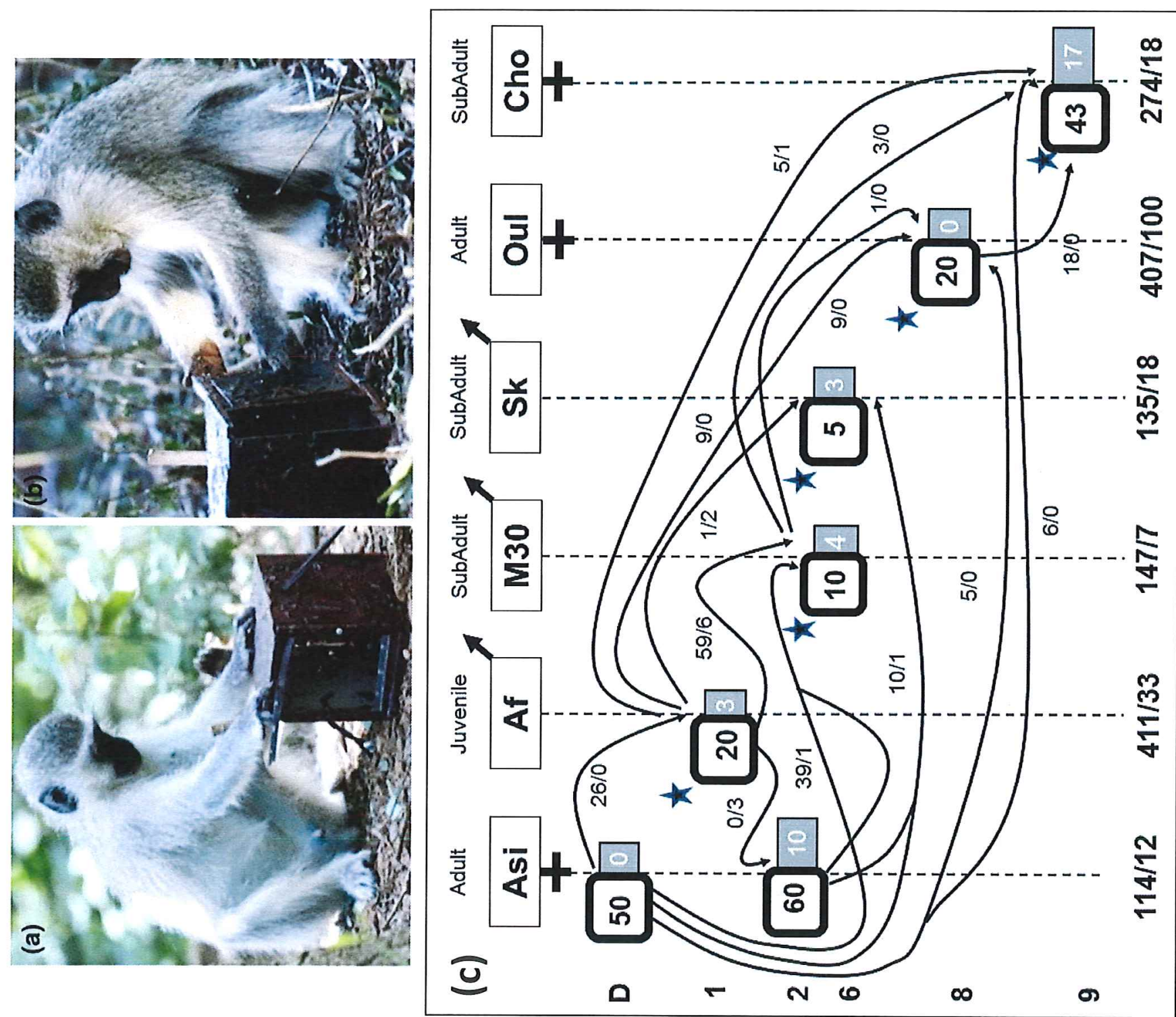
우

()

$\infty$

0

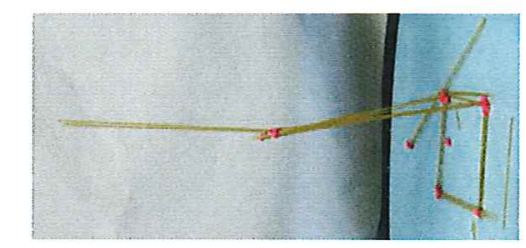

น

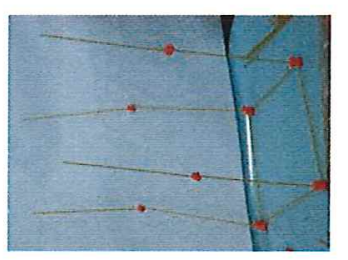

v

m

N

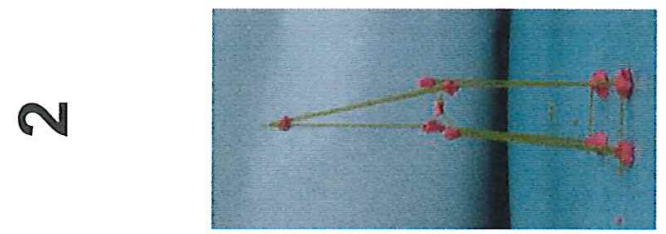

$\nabla$
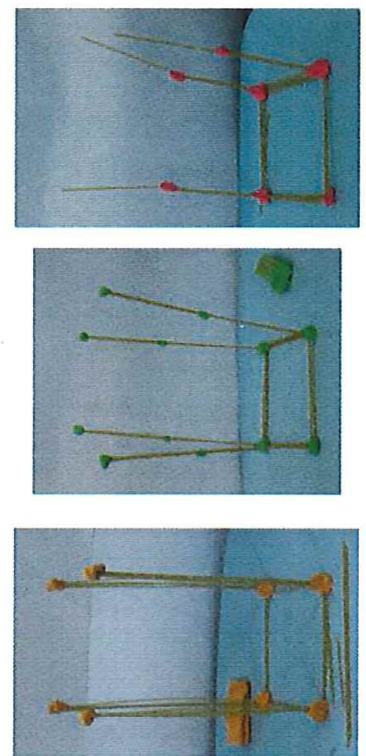

$N$

0
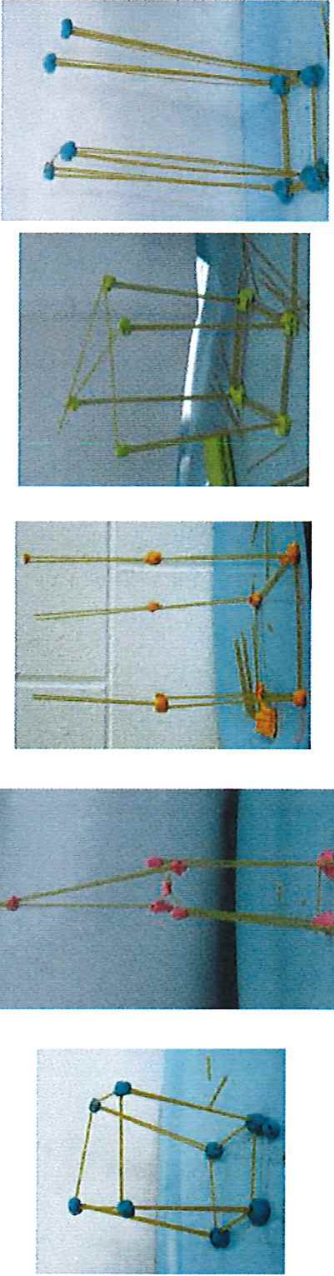
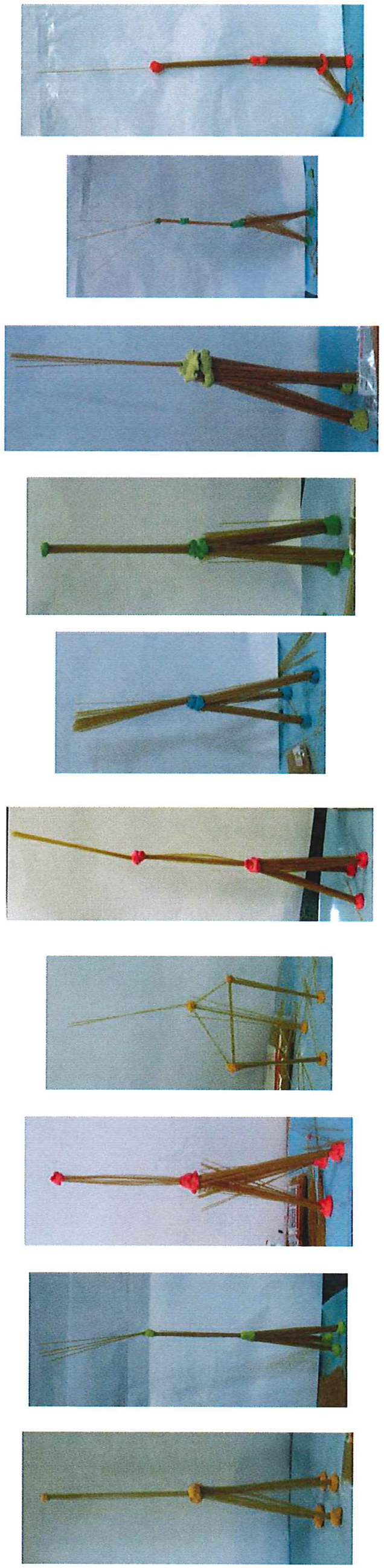


\begin{tabular}{|c|c|c|c|c|c|c|c|c|c|c|}
\hline 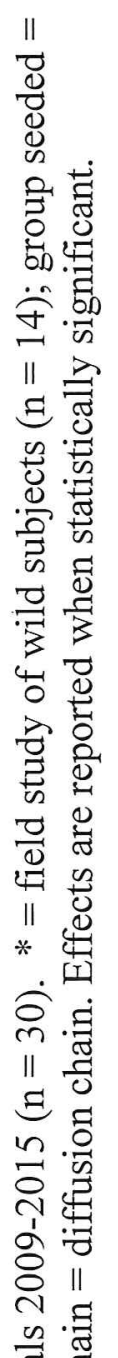 & 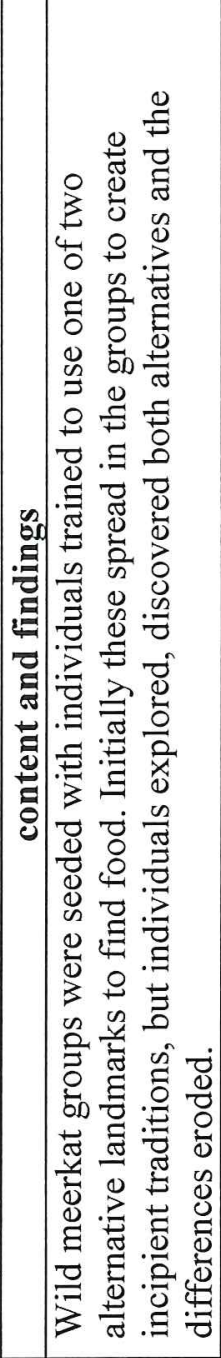 & 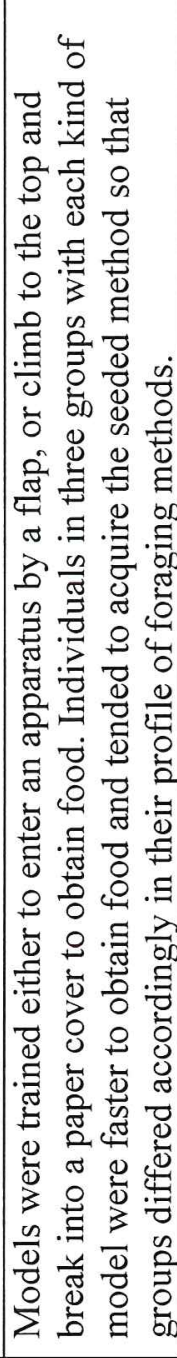 & 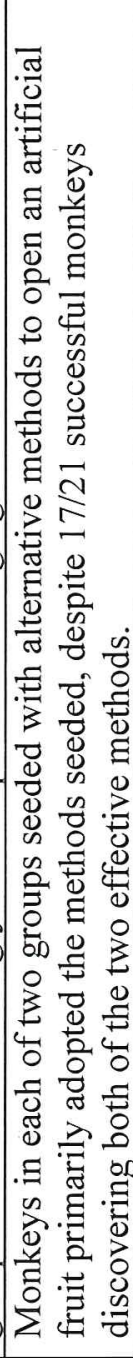 & 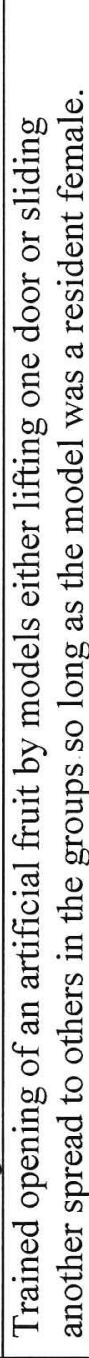 & 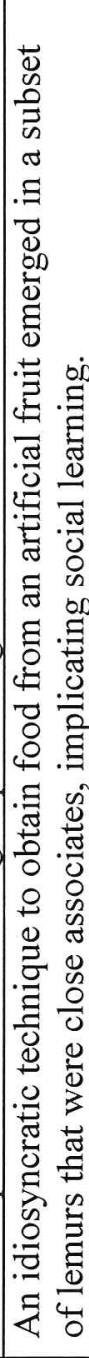 & 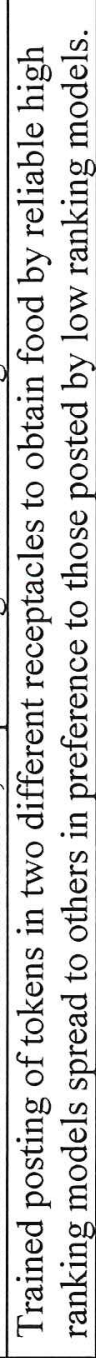 & 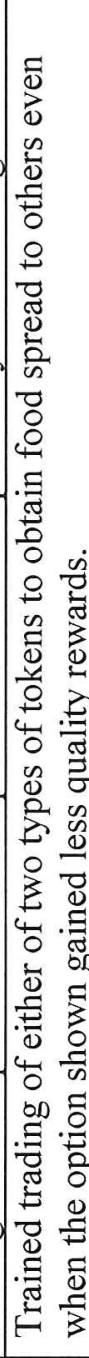 & 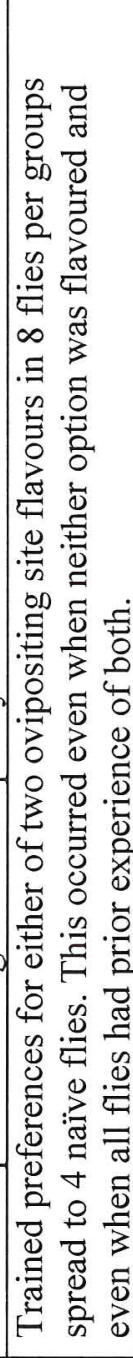 & 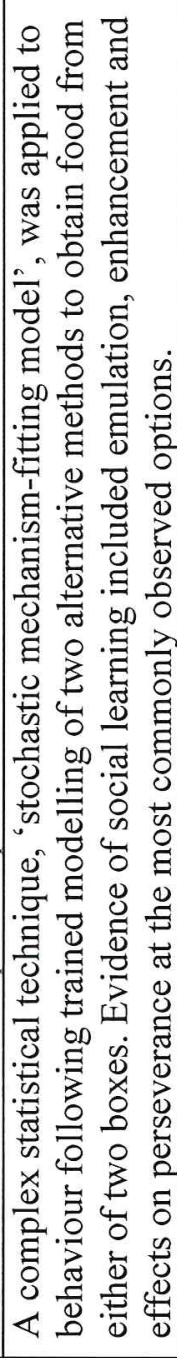 & 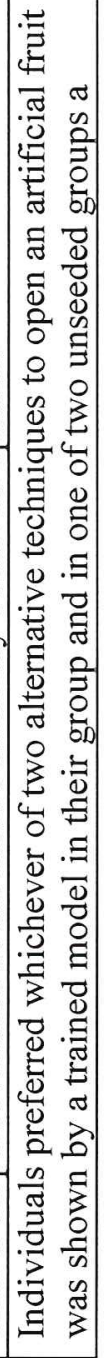 \\
\hline 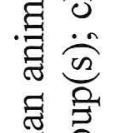 & 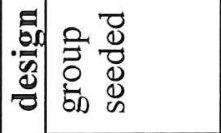 & 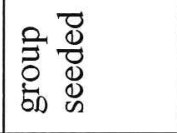 & 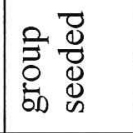 & 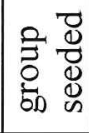 & 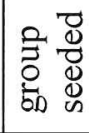 & 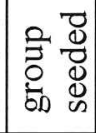 & 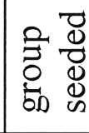 & 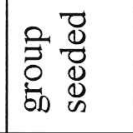 & 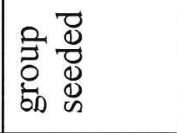 & 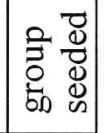 \\
\hline 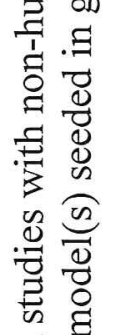 & 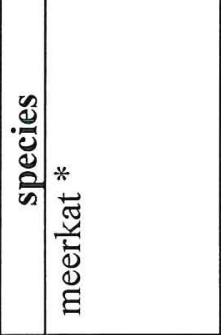 & 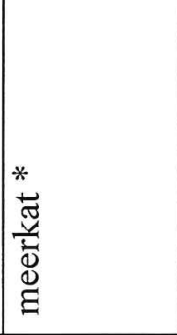 & 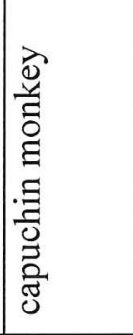 & 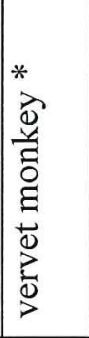 & 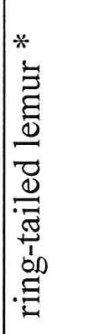 & 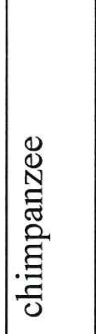 & 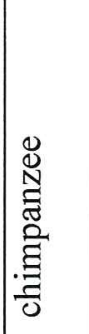 & 戞 & 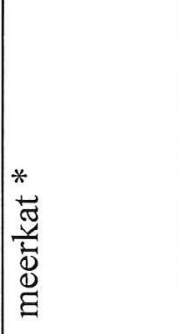 & 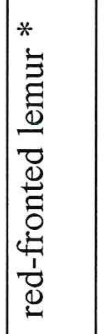 \\
\hline 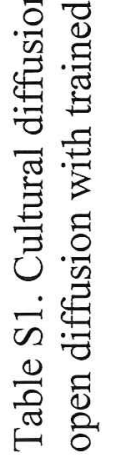 & 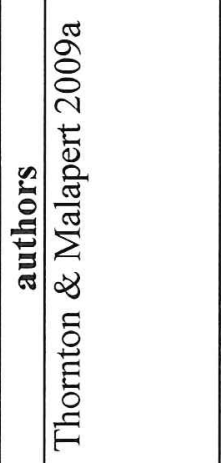 & 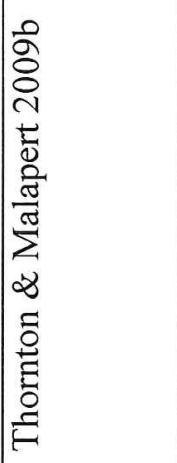 & 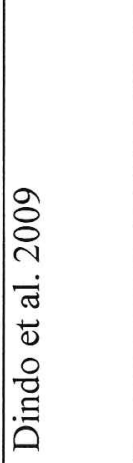 & 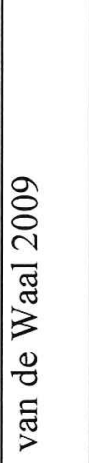 & 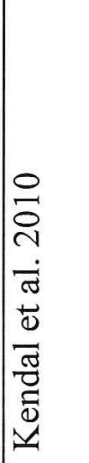 & 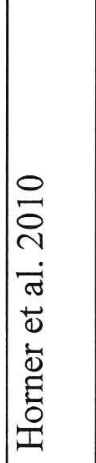 & 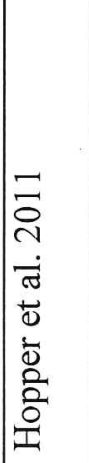 & 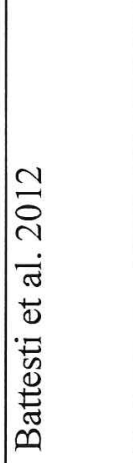 & 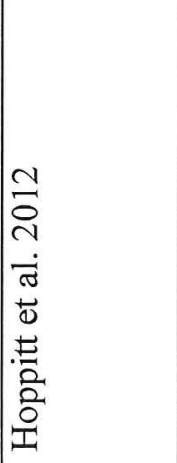 & 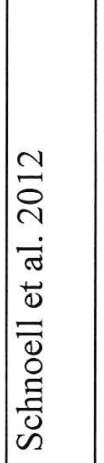 \\
\hline
\end{tabular}




\begin{tabular}{|c|c|c|c|c|c|c|c|c|c|c|}
\hline 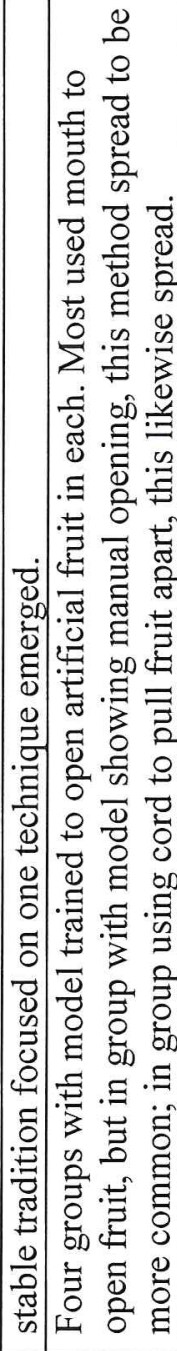 & 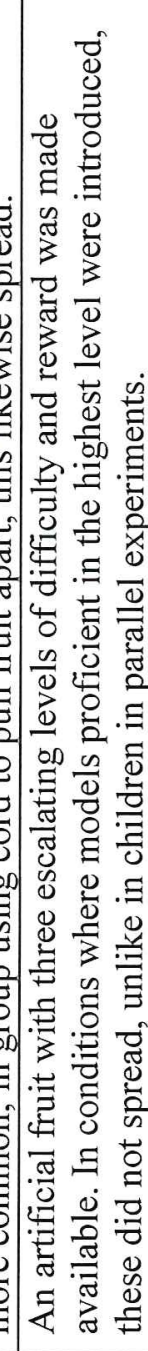 & 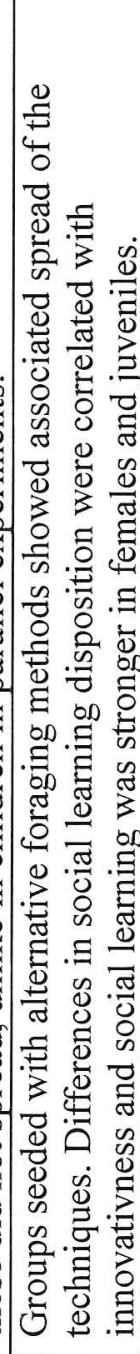 & 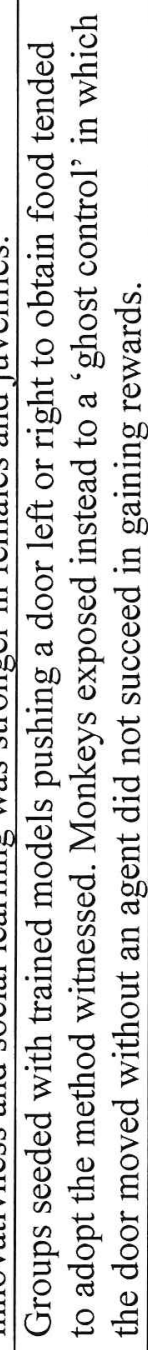 & 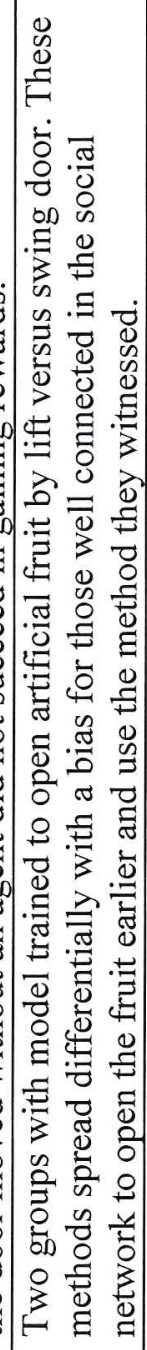 & 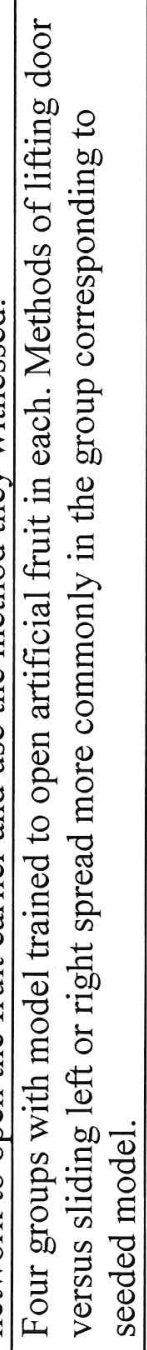 & 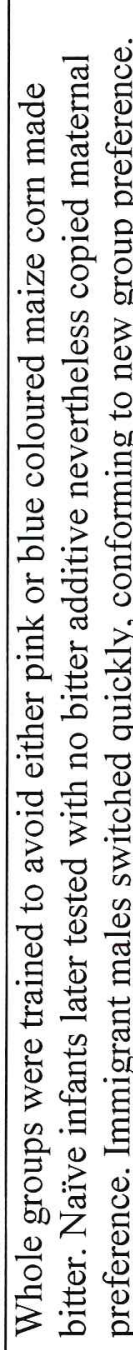 & 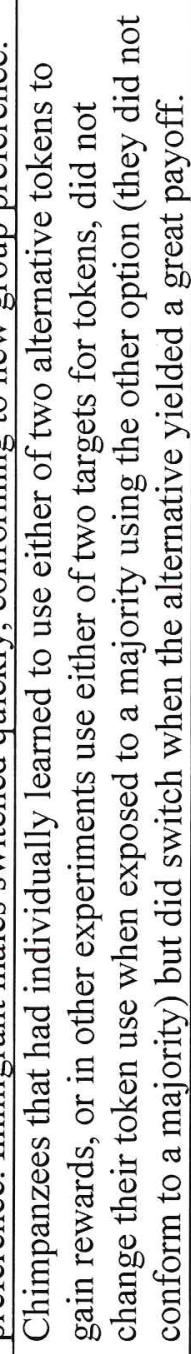 & 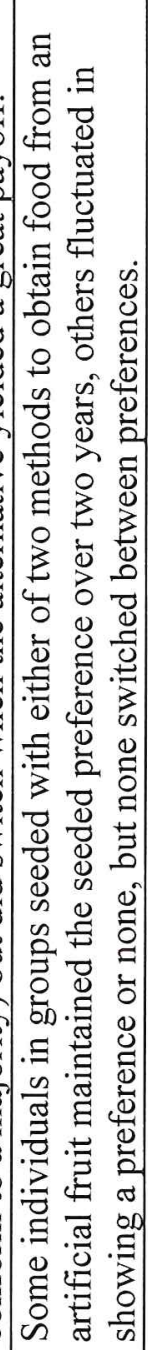 & 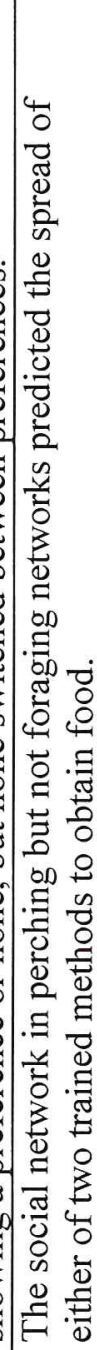 & 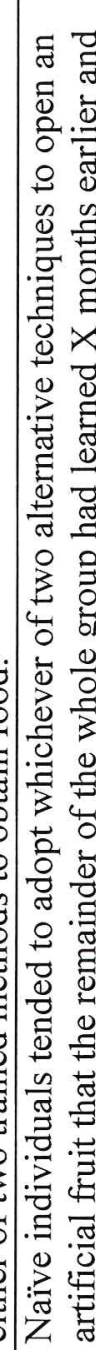 \\
\hline 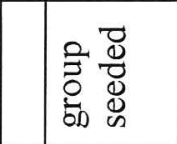 & 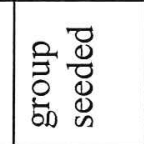 & 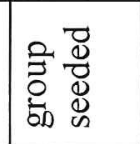 & 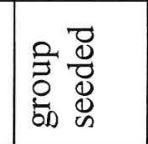 & 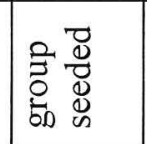 & 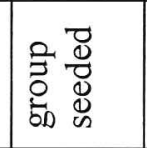 & 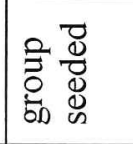 & 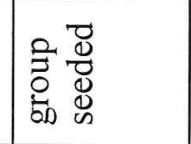 & 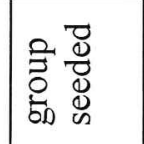 & 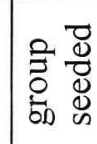 & 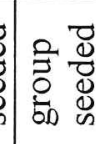 \\
\hline 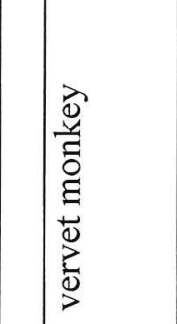 & 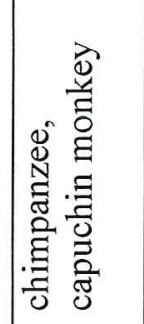 & 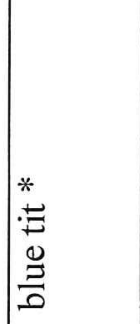 & 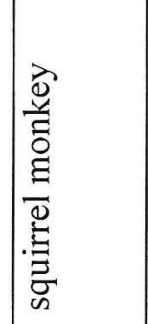 & 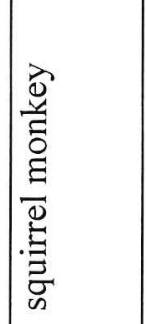 & 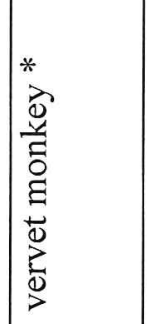 & 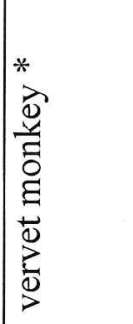 & 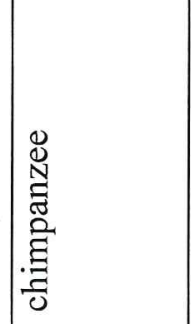 & 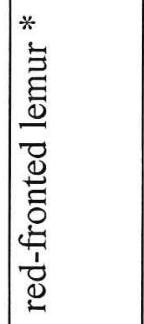 & 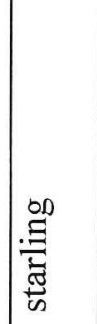 & 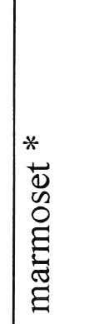 \\
\hline 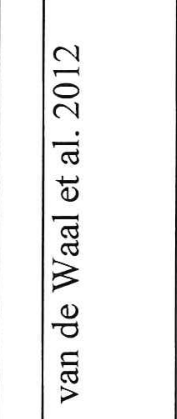 & 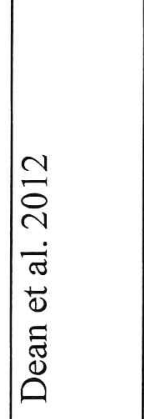 & 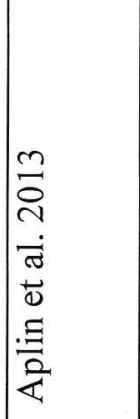 & 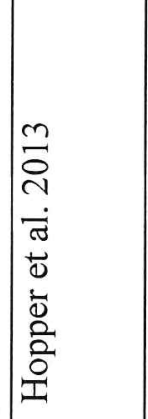 & 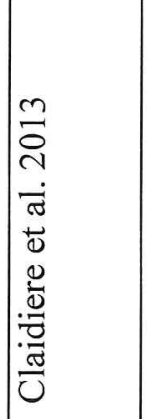 & 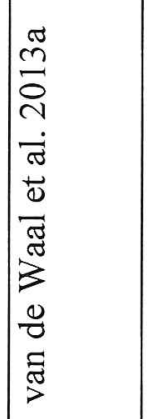 & 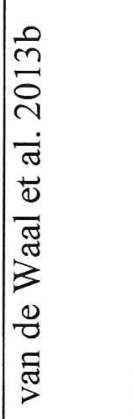 & 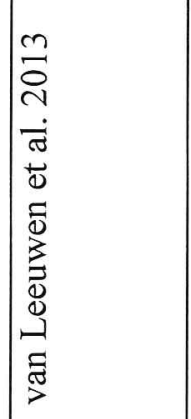 & 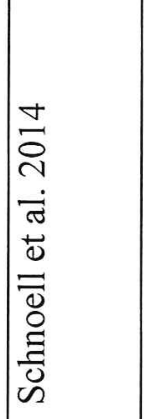 & 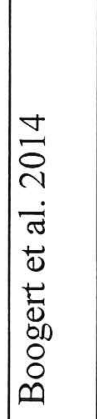 & 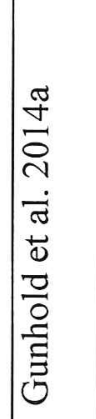 \\
\hline
\end{tabular}




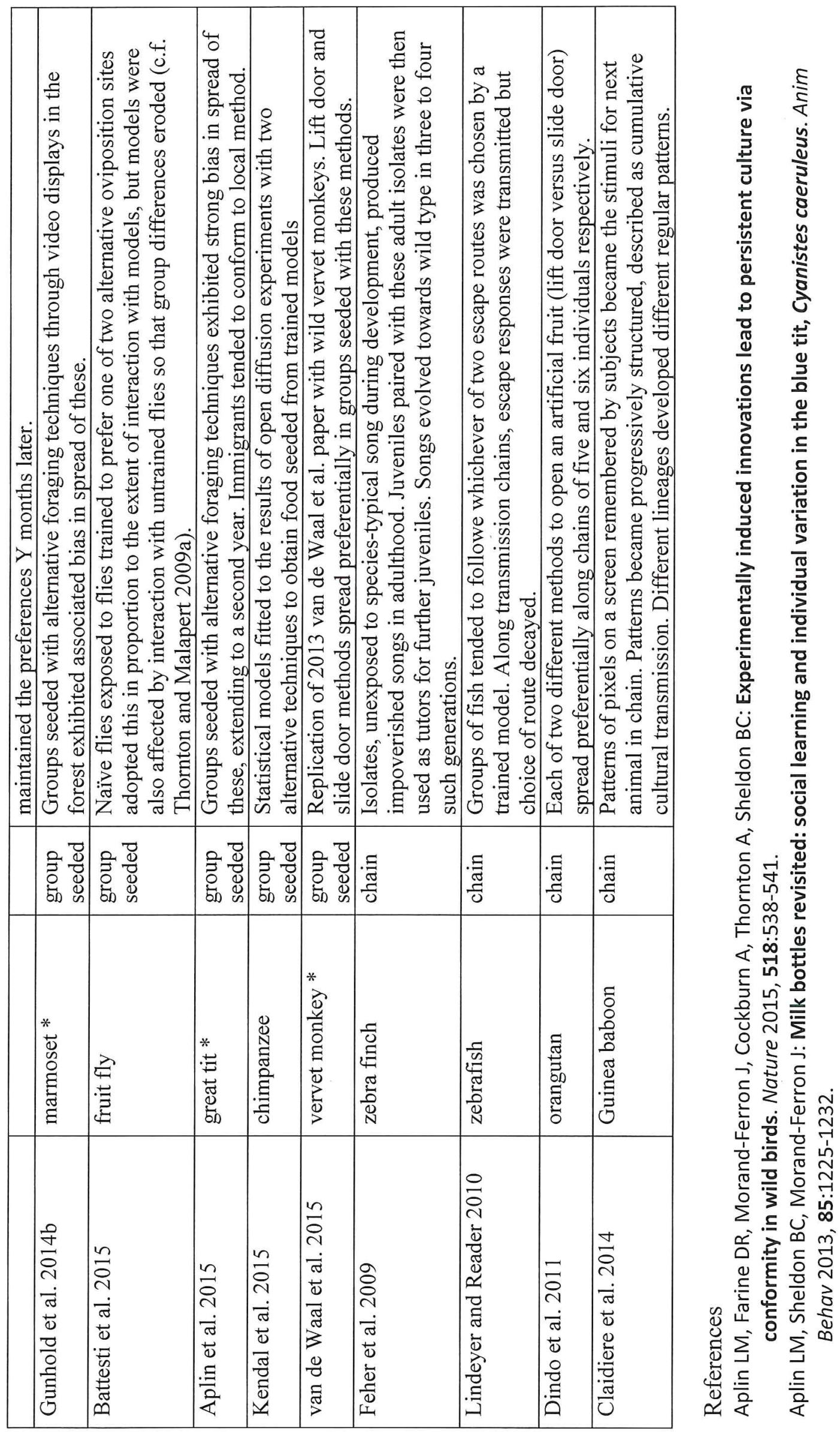




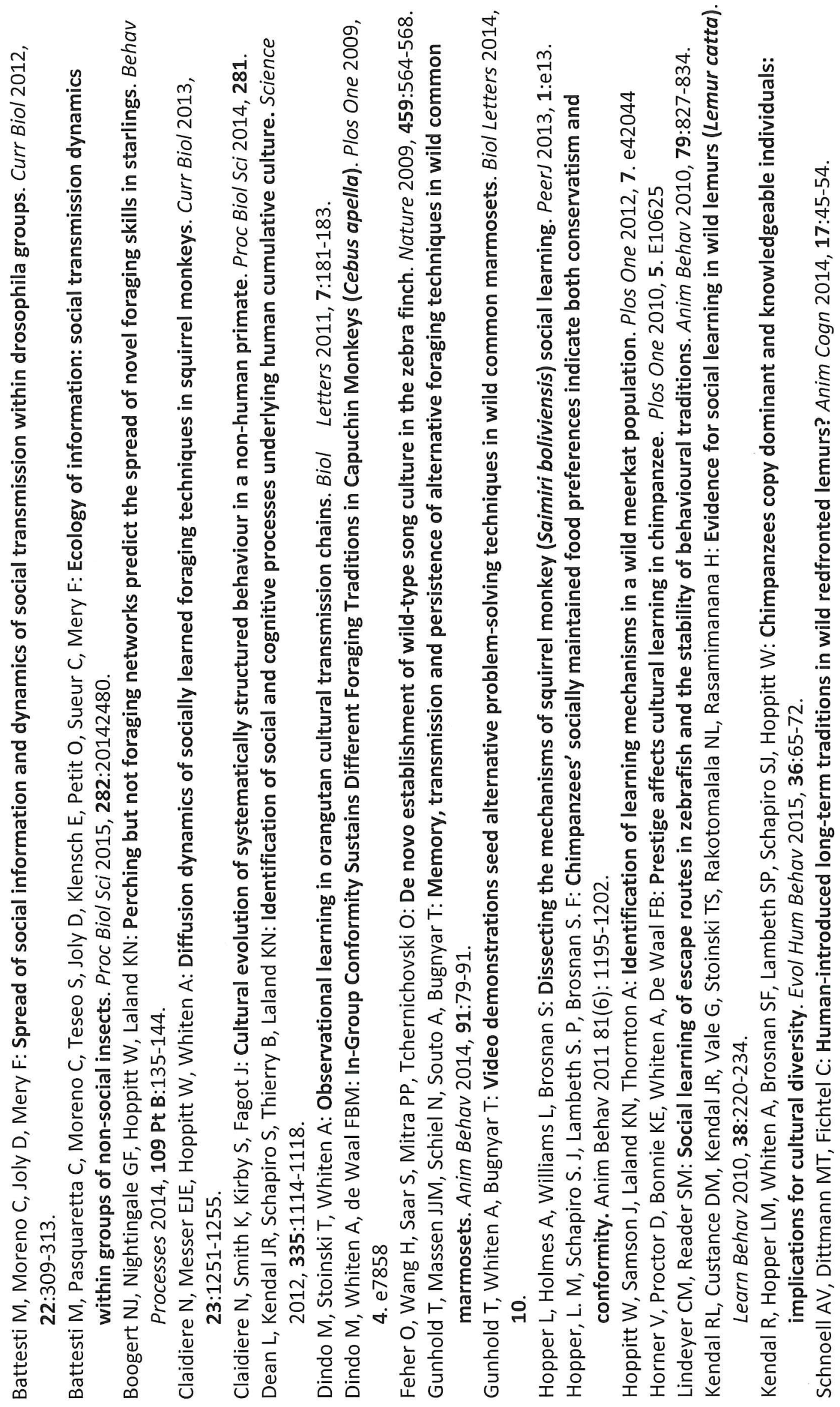




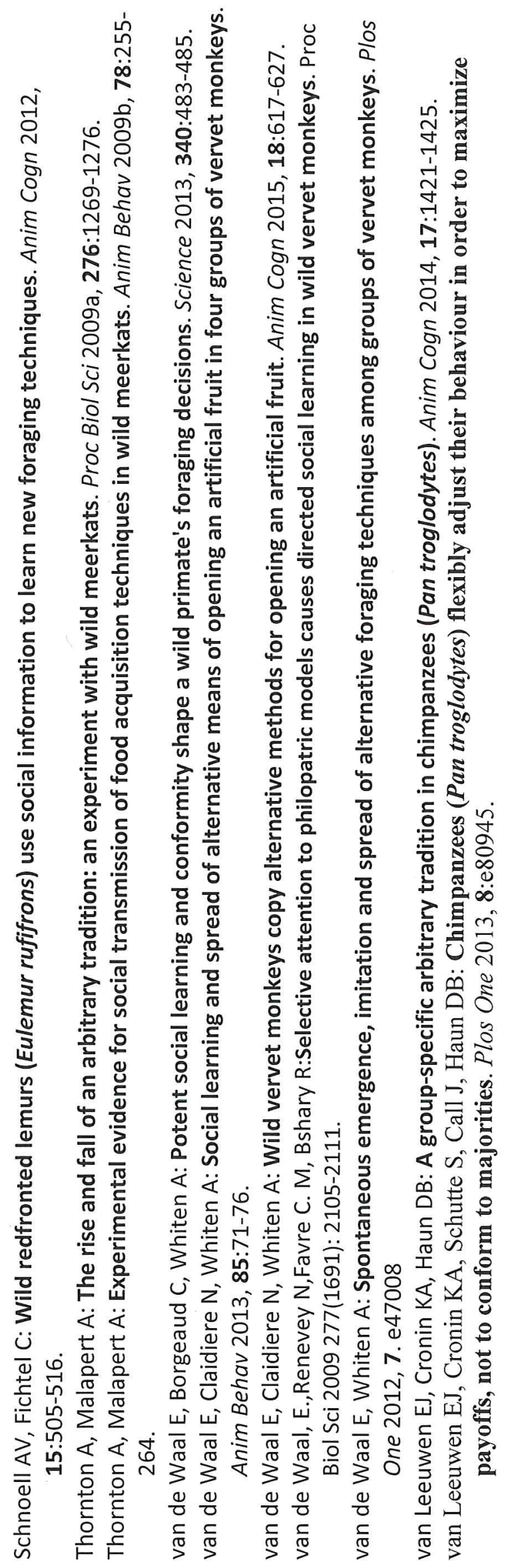




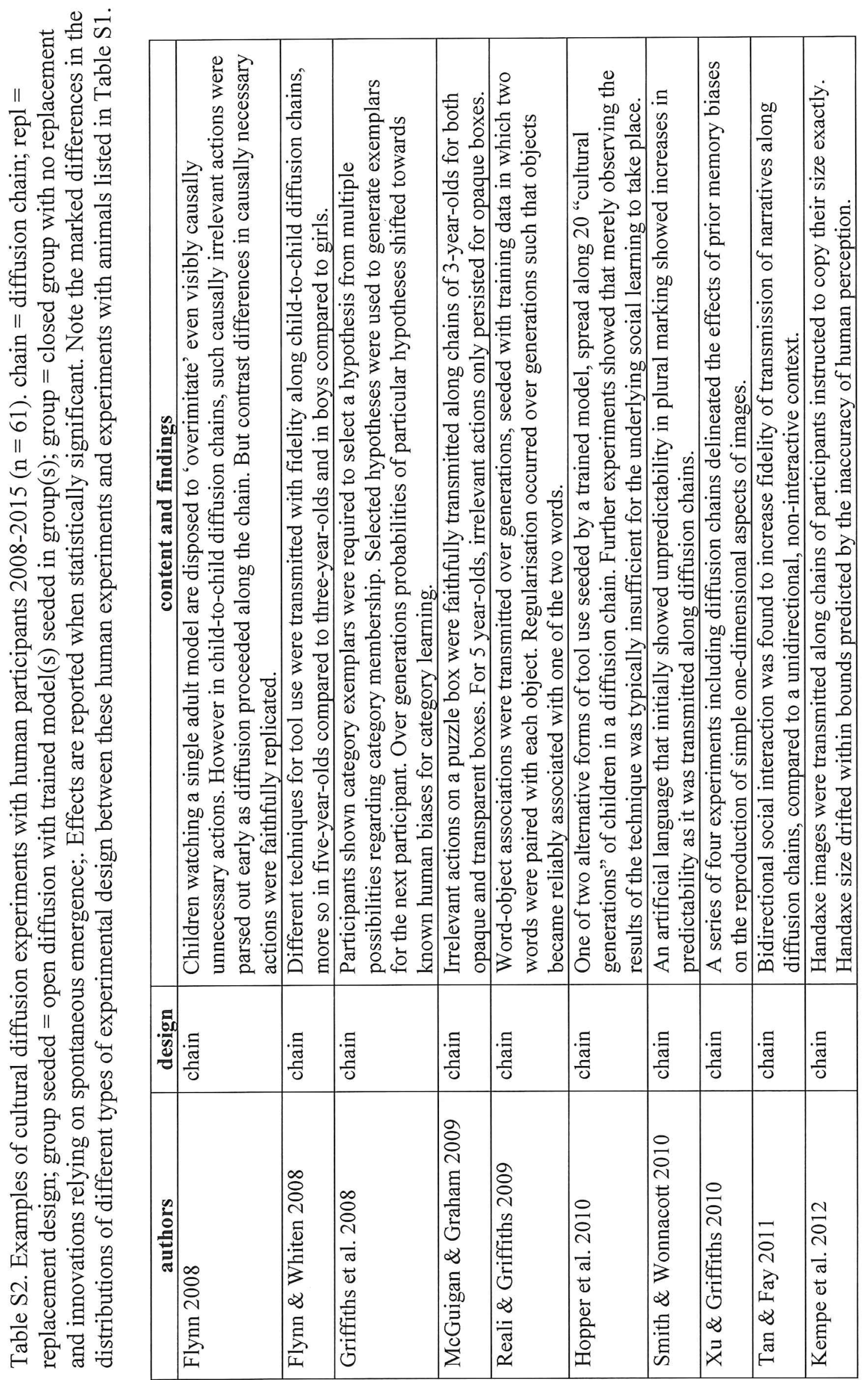




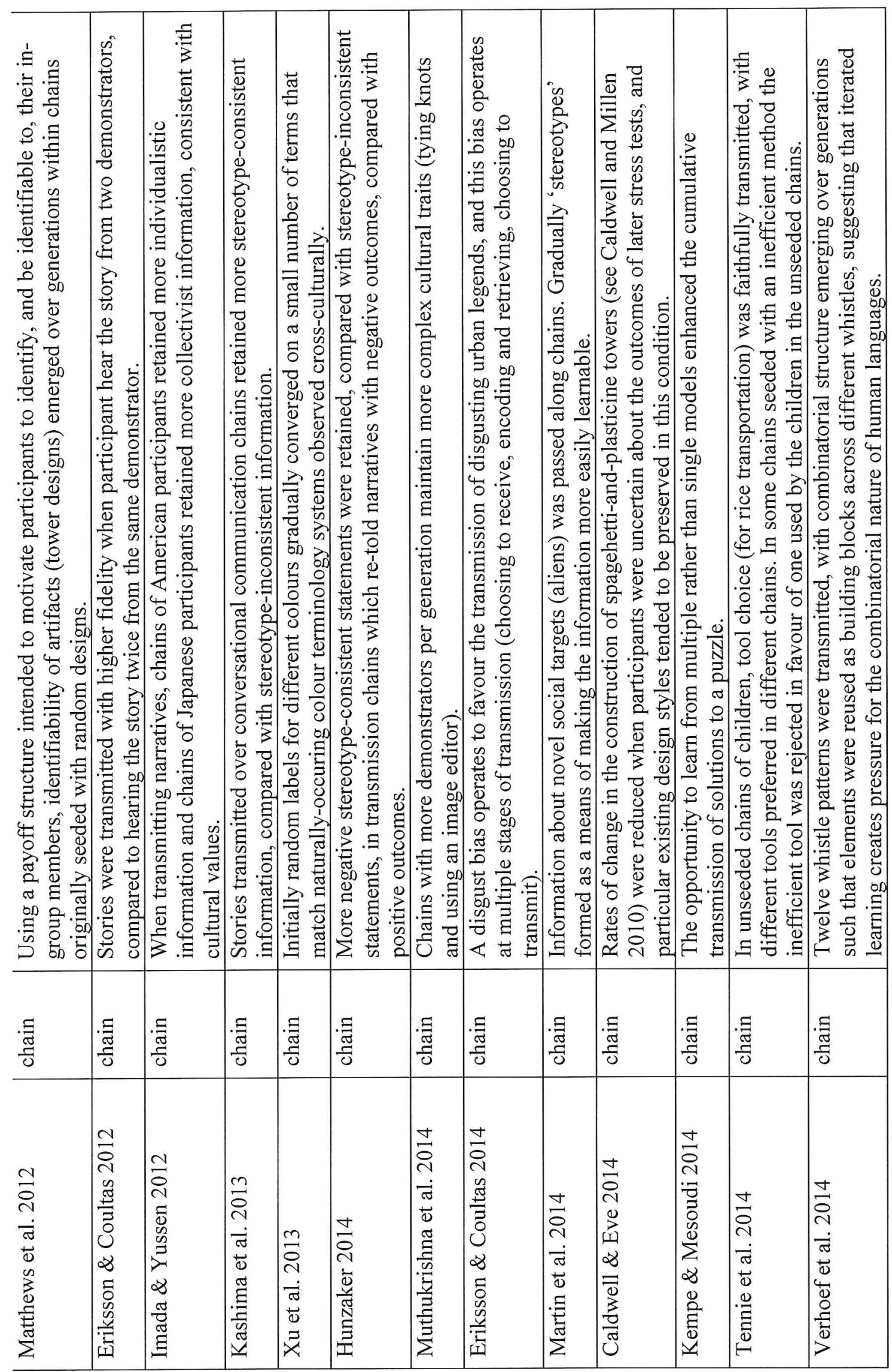




\begin{tabular}{|c|c|c|c|c|c|c|c|c|c|c|c|}
\hline 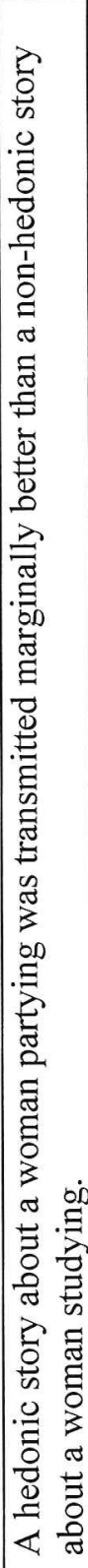 & 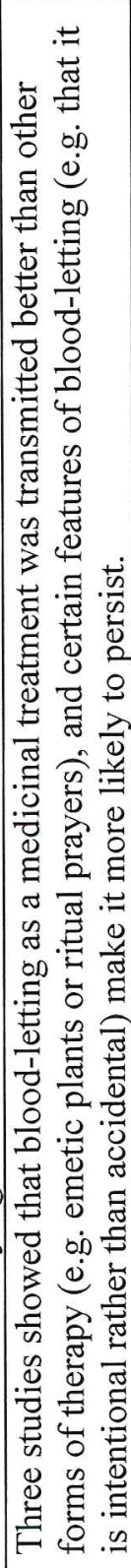 & 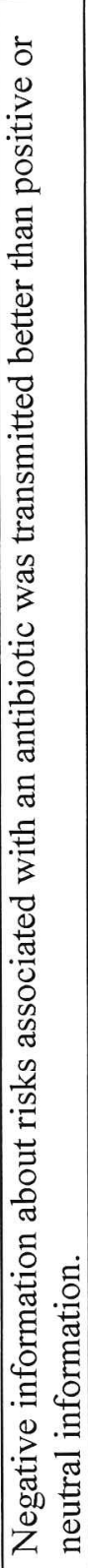 & 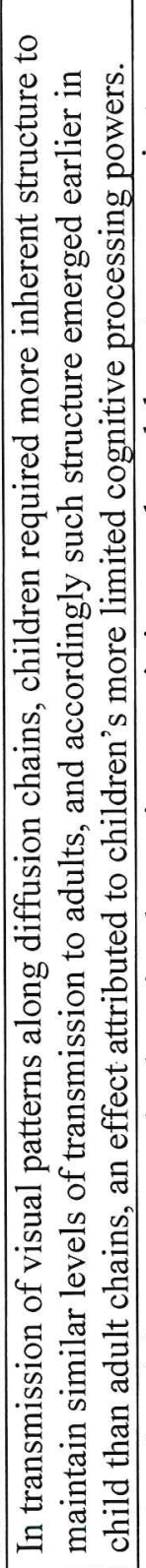 & 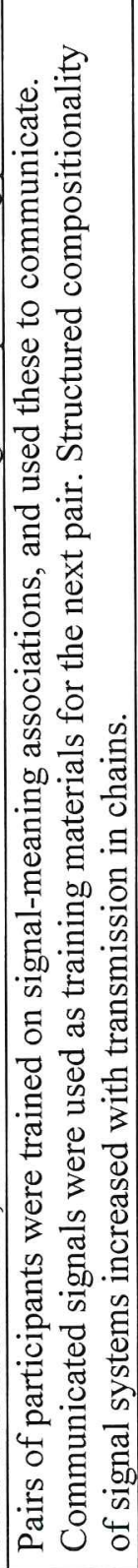 & 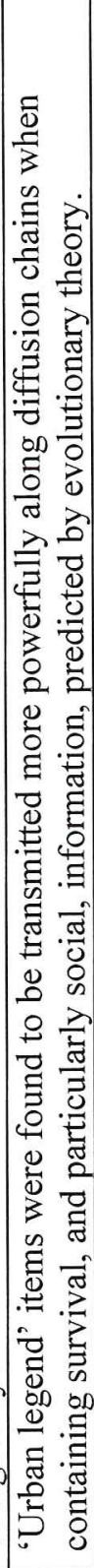 & 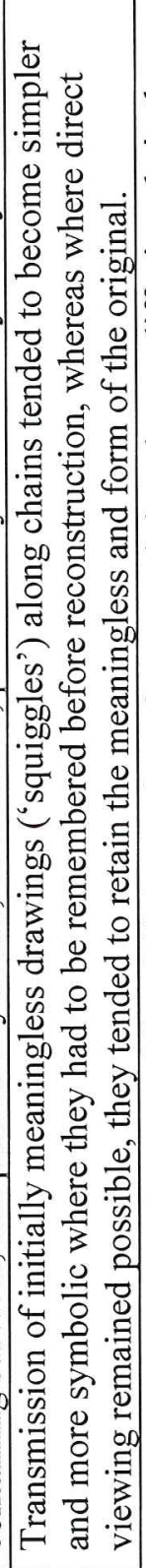 & 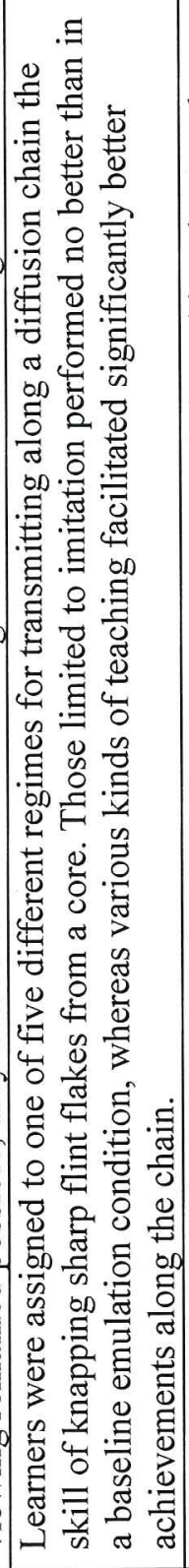 & 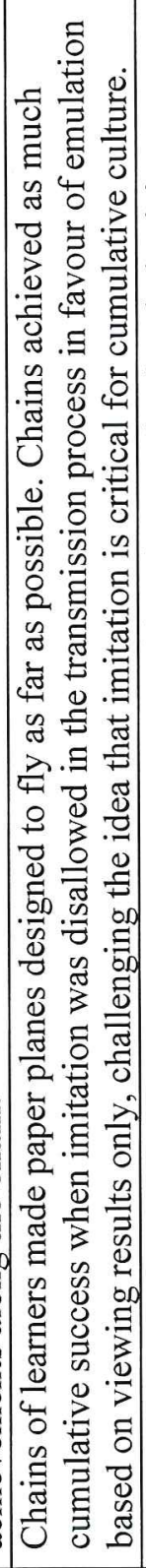 & 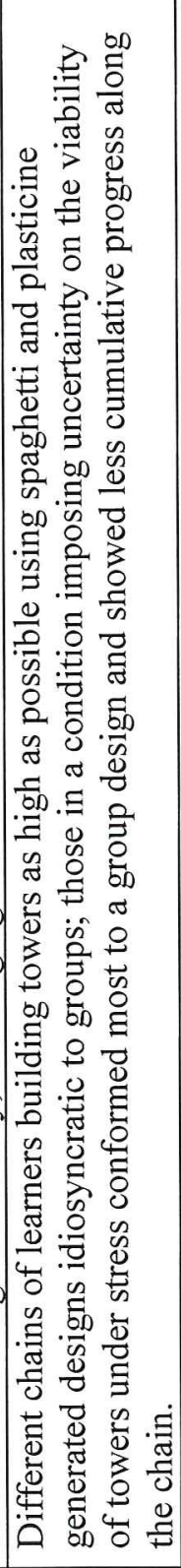 & 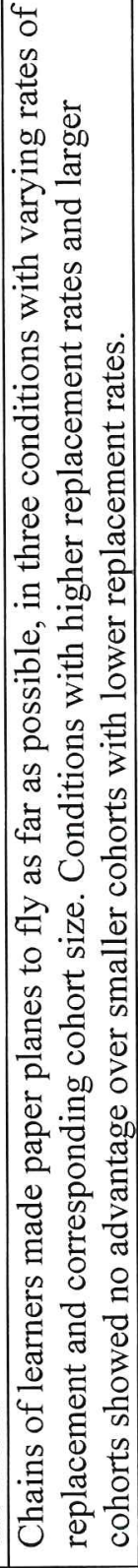 & 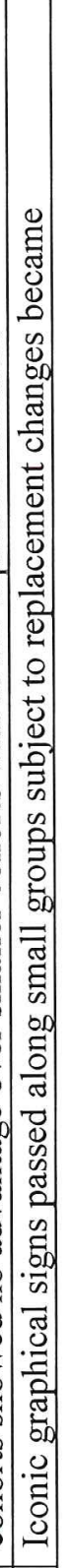 \\
\hline 志 & 离 & 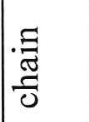 & 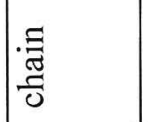 & 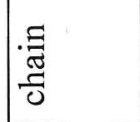 & 䇉 & 丞 & 胥 & $\bar{\partial}$ & $\overrightarrow{0}$ & Qे & $\overrightarrow{0}$ \\
\hline 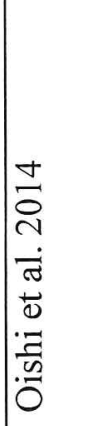 & 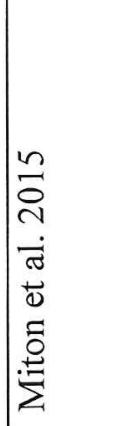 & 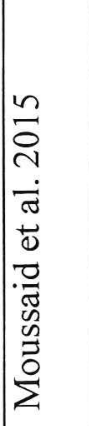 & 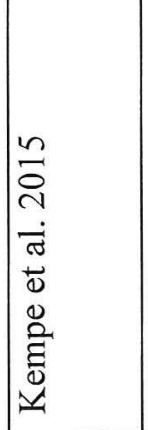 & 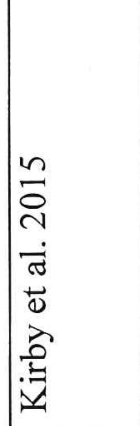 & 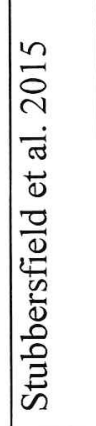 & 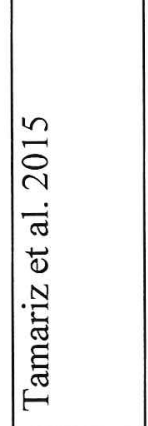 & 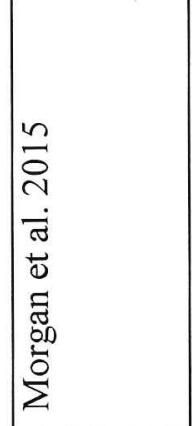 & 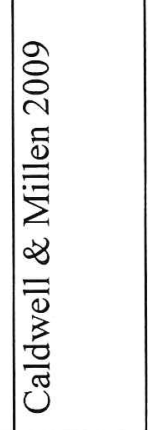 & 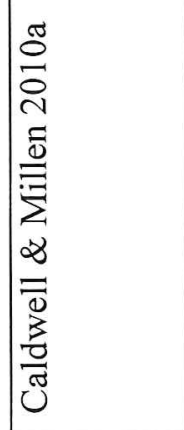 & 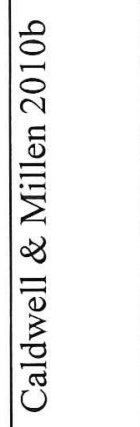 & 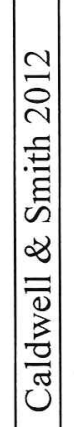 \\
\hline
\end{tabular}




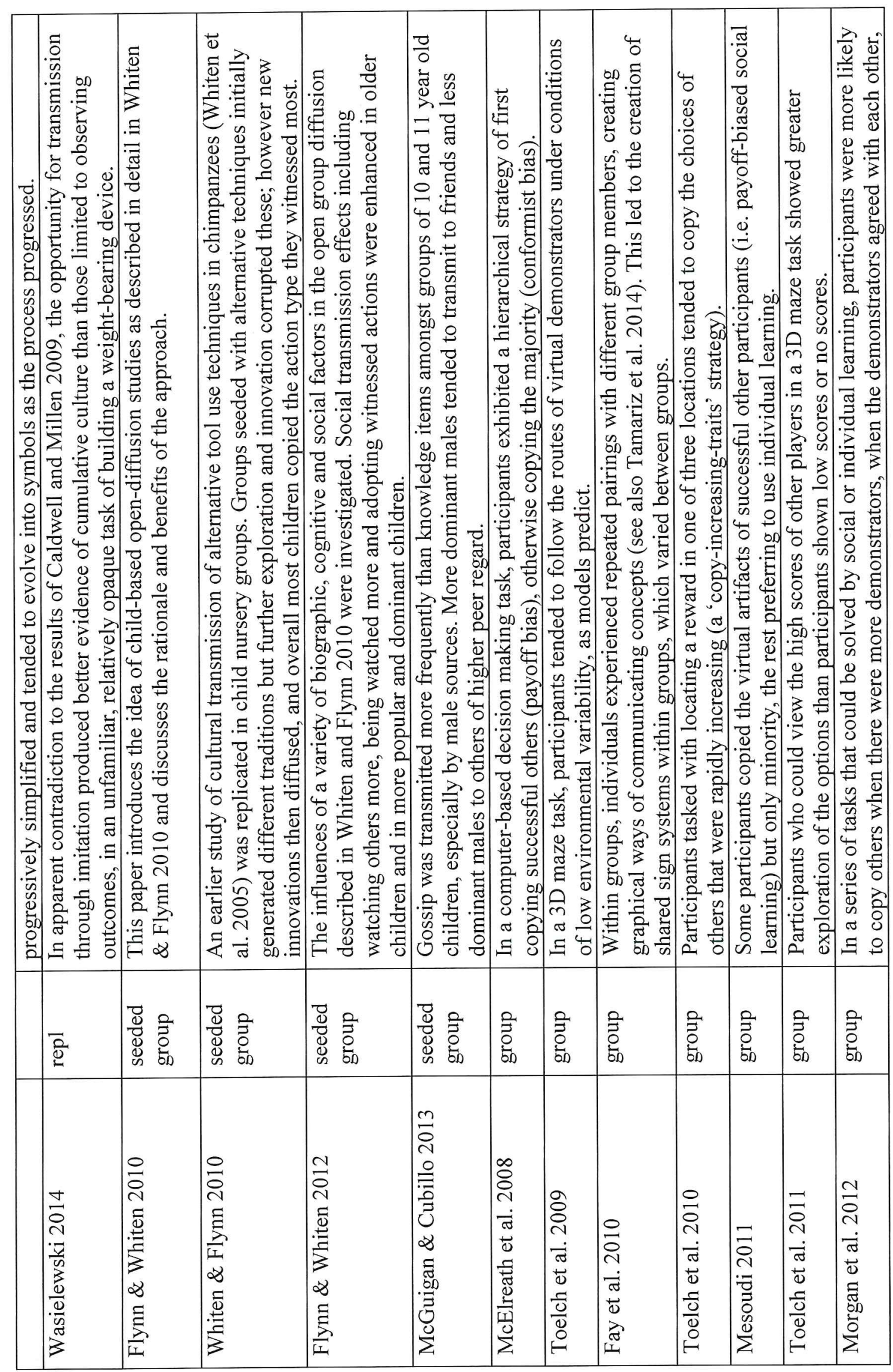




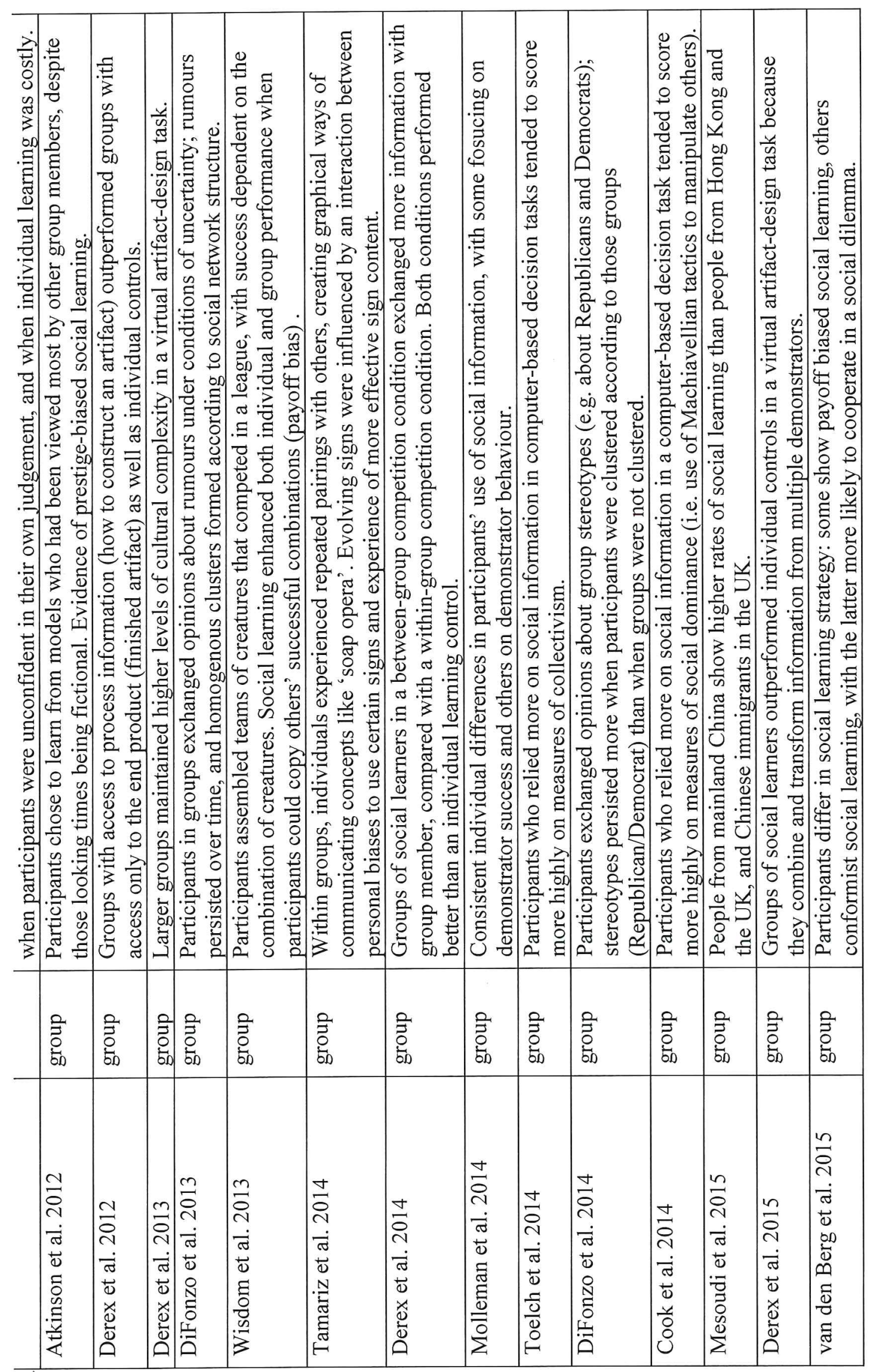




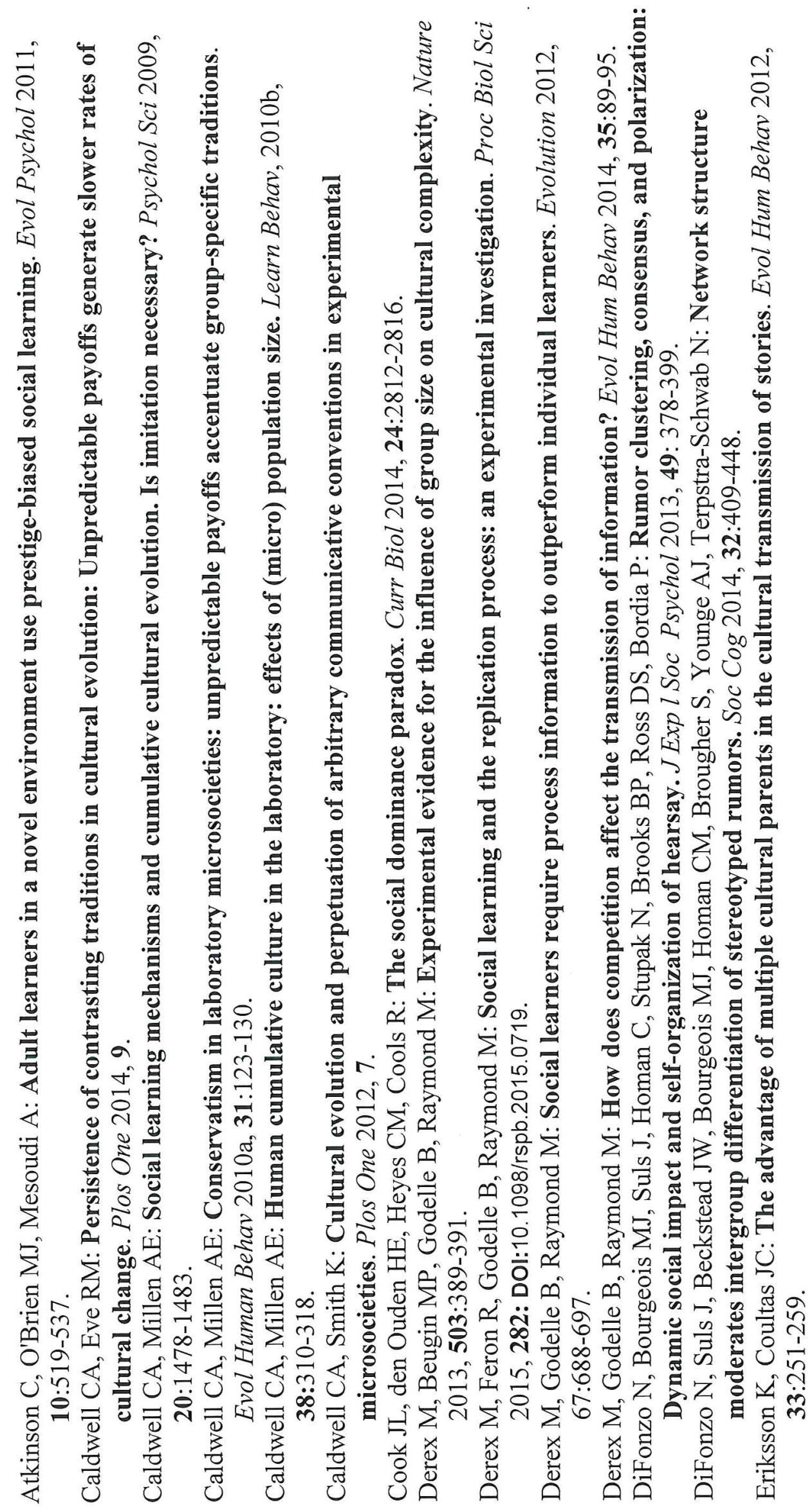




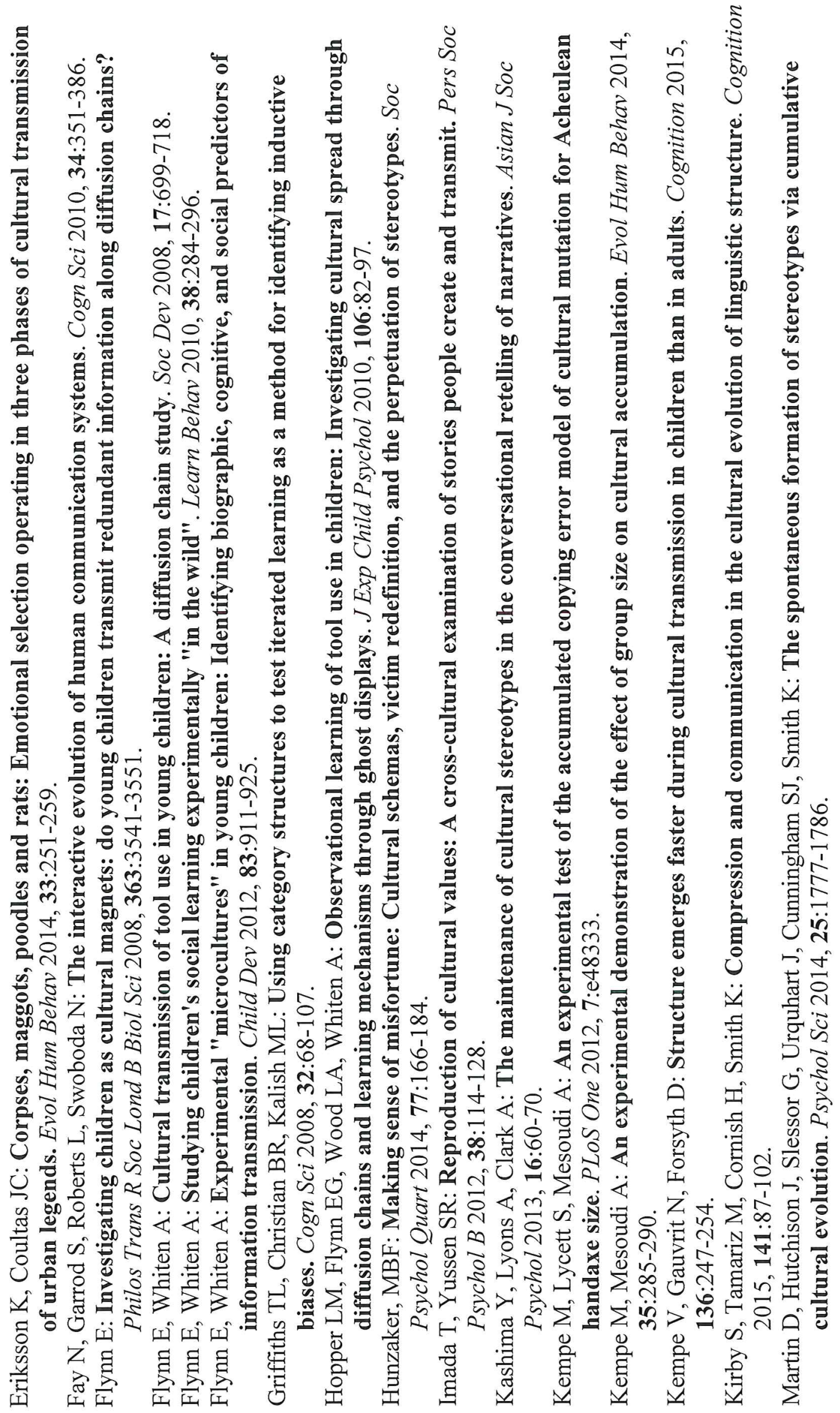




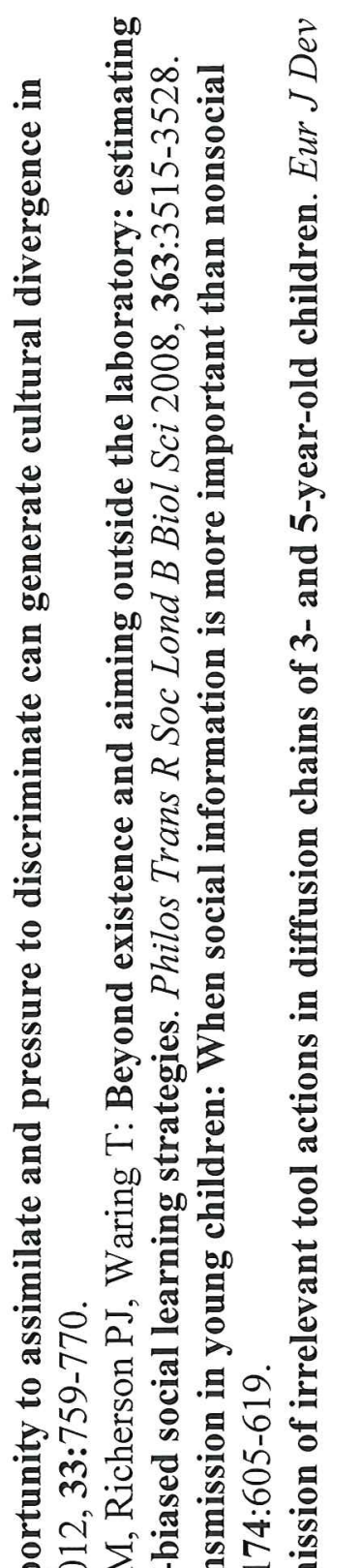

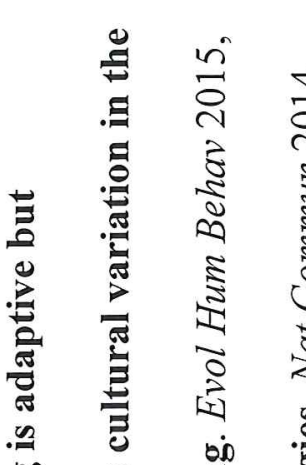

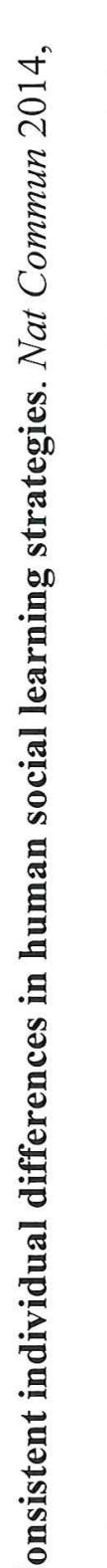

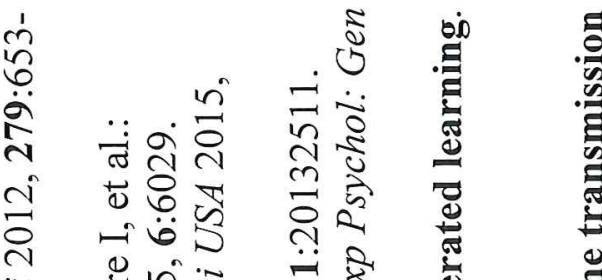

范

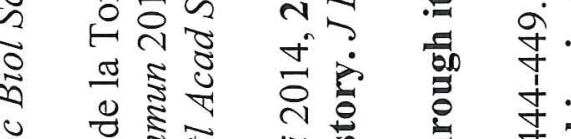

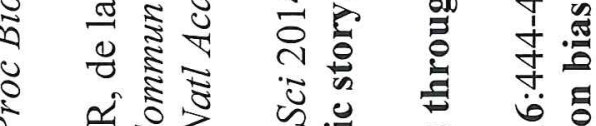

2 वंग

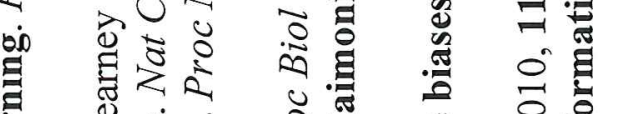

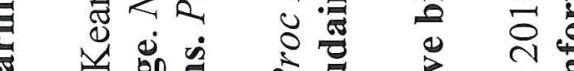

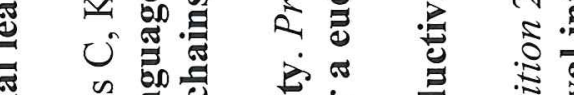

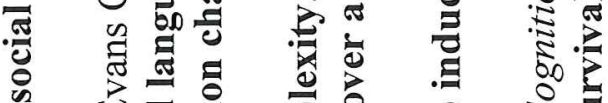

.

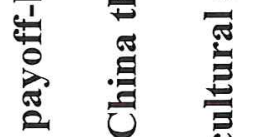

$\ddot{0}$.

क0 000

응

สิ กิ

$\exists$ 궁

담

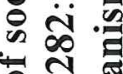

สี

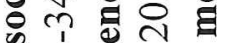

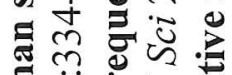

音它 $\widetilde{0}$

है के क्ष

i $\overrightarrow{2}$.

ठิ

论

ํㅛㅇ

ㅇำ

党

哟

$\sum \stackrel{\infty}{0}$

สี

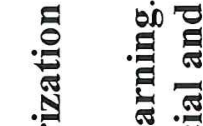

它

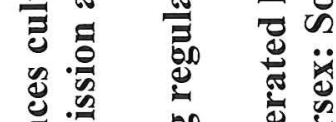

莺

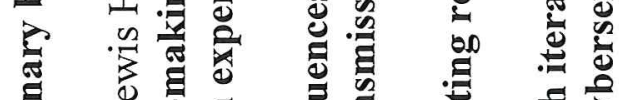

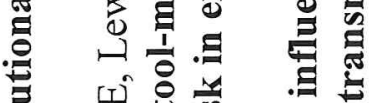

때용

क

㩆 $\infty$

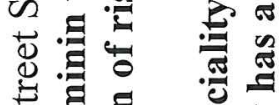

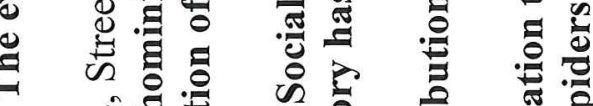

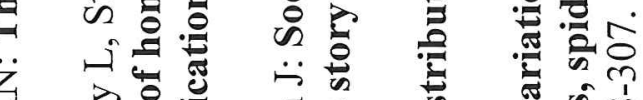

药

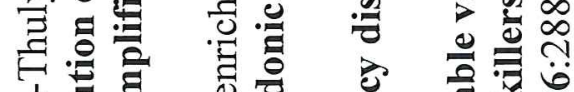

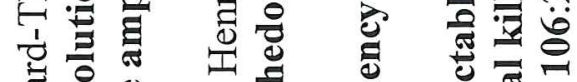

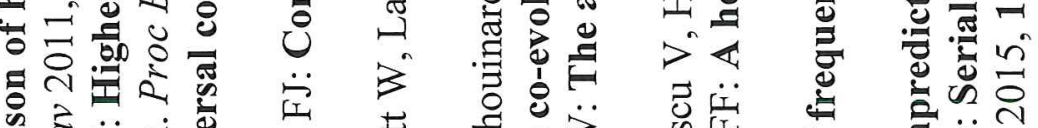

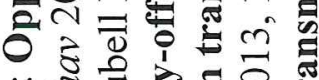

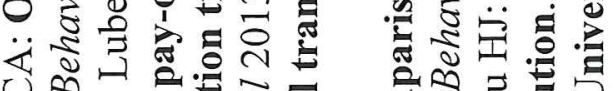

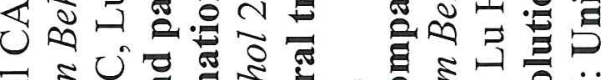

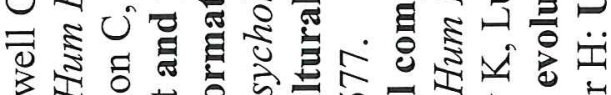

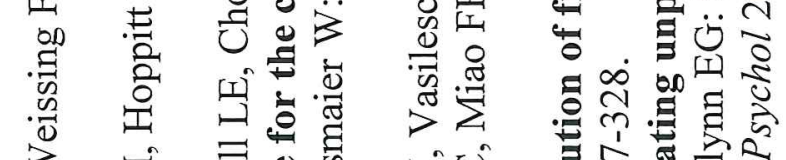

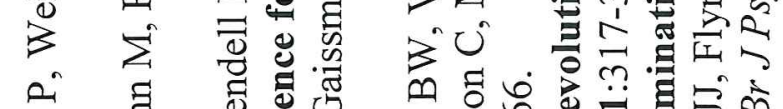

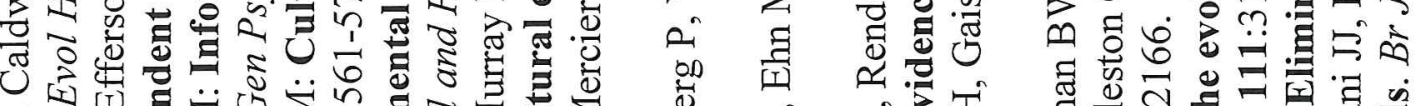

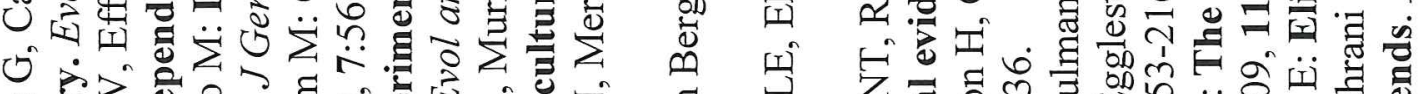

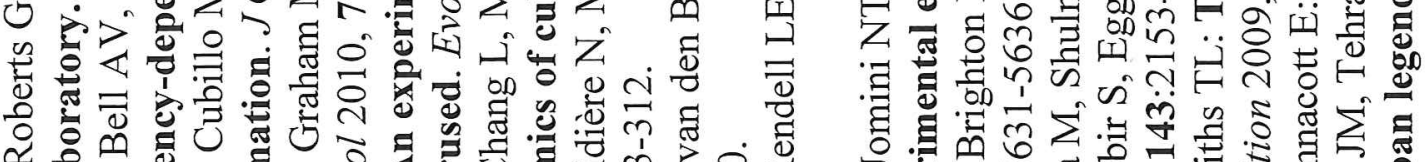
ฯ

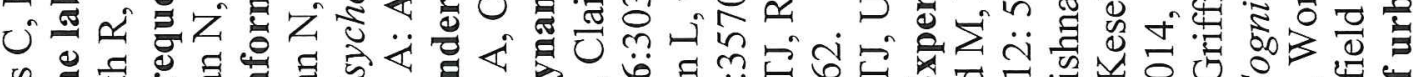

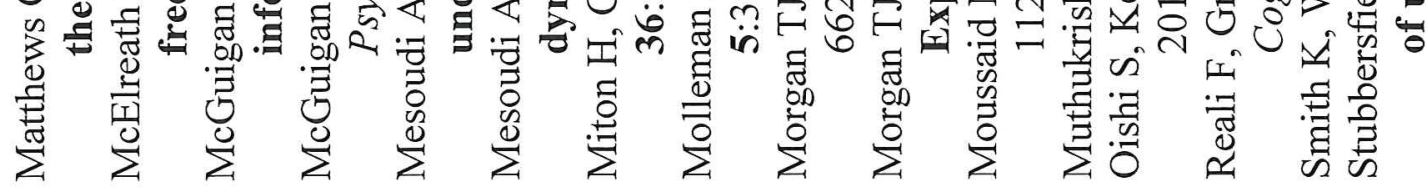




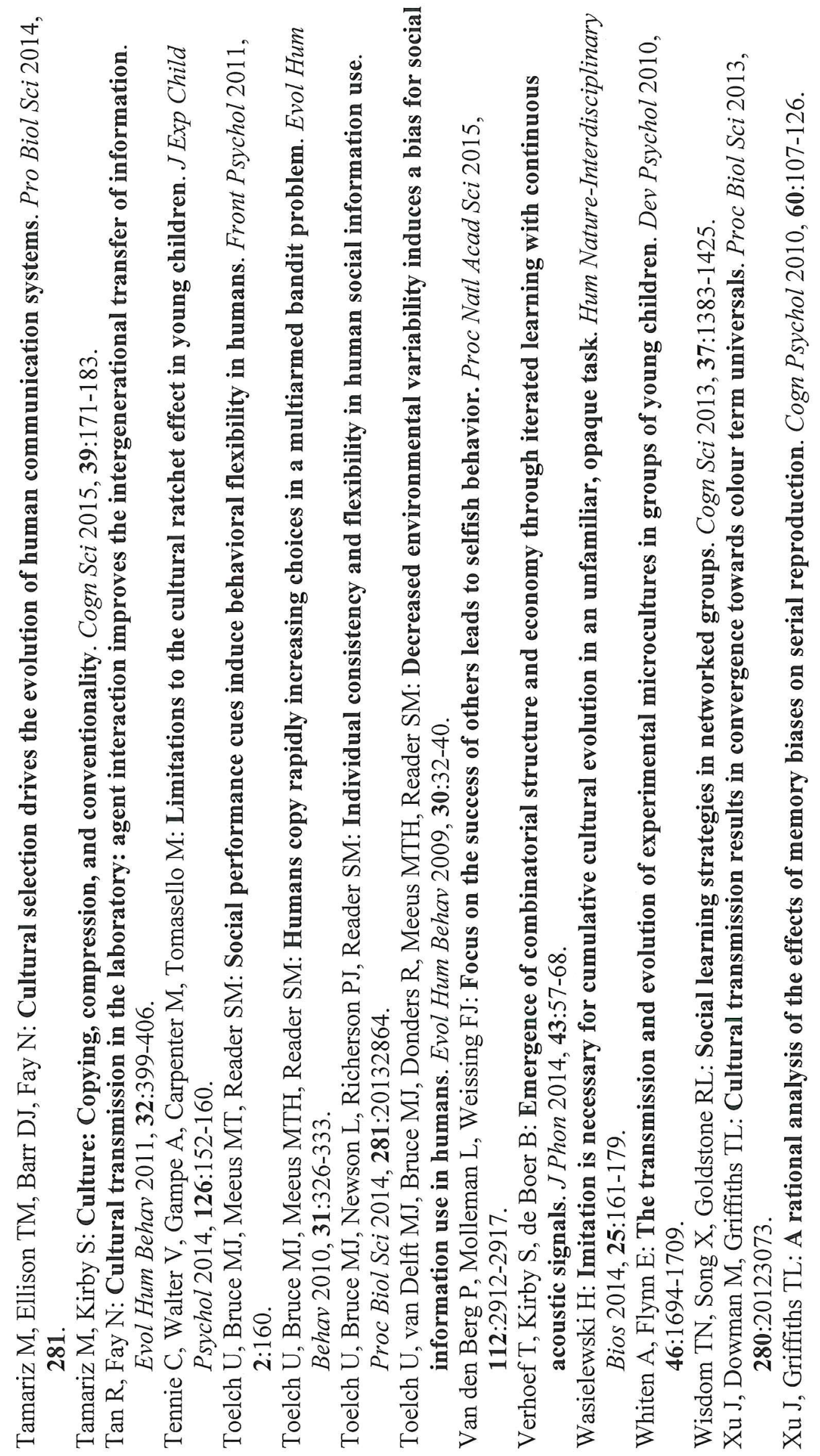

